\title{
CONNECTIONS OF THE MULTIPLE VISUAL CORTICAL AREAS WITH THE LATERAL POSTERIOR-PULVINAR COMPLEX AND ADJACENT THALAMIC NUCLEI IN THE CAT ${ }^{1}$
}

\author{
DENIS RACZKOWSKI ${ }^{2}$ AND ALAN C. ROSENQUIST ${ }^{3}$ \\ Department of Anatomy, University of Pennsylvania, Philadelphia, Pennsylvania 19104
}

Received September 17, 1982; Revised February 24, 1983; Accepted March 3, 1983

\begin{abstract}
The present report describes the patterns of cat thalamocortical interconnections for each of the 13 retinotopically ordered visual areas and additional visual areas for which no retinotopy has yet emerged. Small injections $(75 \mathrm{nl})$ of a mixture of horseradish peroxidase and $\left[{ }^{3} \mathrm{H}\right]$ leucine were made through a recording pipette at cortical injection sites identified by retinotopic mapping. 'T'he patterns of thalamic label show that the lateral posterior-pulvinar complex of the cat is divided into three distinct functional zones, each of which contains a representation of the visual hemifield and shows unique afferent and efferent connectivity patterns. The pulvinar nucleus projects to areas $19,20 \mathrm{a}$, $20 \mathrm{~b}, 21 \mathrm{a}, 21 \mathrm{~b}, 5,7$, the splenial visual area, and the cingulate gyrus. The lateral division of the lateral posterior nucleus projects to areas $17,18,19,20 \mathrm{a}, 20 \mathrm{~b}, 21 \mathrm{a}, 21 \mathrm{~b}$, and the anterior medial (AMLS), posterior medial (PMLS), and ventral (VLS) lateral suprasylvian areas. The medial division of the lateral posterior nucleus projects to areas AMLS, PMLS, VLS, and the anterior lateral (ALLS), posterior lateral (PLLS), dorsal (DLS) lateral suprasylvian areas, and the posterior suprasylvian areas. In addition, many of these visual areas are also interconnected with subdivisions of the dorsal lateral geniculate nucleus (LGd). Every retinotopically ordered cortical area (except ALLS and AMLS) is reciprocally interconnected with the parvocellular C layers of the LGd. The medial intralaminar nucleus of the LGd projects to areas 17, 18, 19, AMLS, and PMLS. Finally, each cortical area (except area 17) receives a projection from thalamic intralaminar nuclei. These results help to define the pathways by which visual information gains access to the vast system of extrastriate cortex in the cat.
\end{abstract}

The mammalian visual system is no longer viewed solely as a serial chain of processing stations from the retina through the dorsal lateral geniculate nucleus $\left(\mathrm{LGd}^{1}\right)$ to striate and then to extrastriate cortex. Instead,

\footnotetext{
${ }^{1}$ We dedicate this paper to Professors Irving T. Diamond and James M. Sprague for their pioneering contributions to the concept of parallel visual pathways. This work was supported by National Institutes of Health Grants EY 02654, EY 05342, EY 07035, and EY 01583. We wish to thank Jeanne Levy for technical assistance, Theresa Grigsby for secretarial assistance, as well as John and Bette Woolsey, Tom Sarrantonio, and Pauline Wong for preparation of the illustrations. Drs. Mark Segraves, Elin Kaufman, and Laura Symonds participated in many of the experiments described in this paper.

${ }^{2}$ Present address: Department of Neurobiology and Behavior, SUNY, Stony Brook, NY 11794.

${ }^{3}$ To whom correspondence should be addressed, at Department of Anatomy, University of Pennsylvania, 36th and Hamilton Walk, Philadelphia, PA 19104.

${ }^{4}$ The abbreviations used are: ALLS, anterior lateral lateral suprasylvian area; AMLS, anterior medial lateral suprasylvian area; CM,
}

a number of parallel pathways are now recognized by which visual information is relayed through both the geniculate and extrageniculate visual thalamus to multiple areas of the visual cortex (Diamond and Hall, 1969; Diamond, 1973, 1976; Rodieck, 1979; Van Essen, 1979). The fact that extrastriate cortical areas function importantly for visual perception even in the absence of striate cortex underscores the functional significance of these additional "extrageniculate" visual pathways (Killackey et al., 1971; Sprague et al., 1977; Berkley and Sprague, 1979). In the cat, the major extrageniculate way-station

central medial nucleus; CL, central lateral nucleus; DLS, dorsal lateral suprasylvian area; HM, horizontal meridian; LGd, dorsal lateral geniculate nucleus; $\mathrm{LP}$, lateral posterior nucleus ( $\mathrm{LPl}$ and $\mathrm{LPm}) ; \mathrm{LPl}$, lateral division of lateral posterior nucleus; LPm, medial division of lateral posterior nucleus; MIN, medial interlaminar nucleus; PLLS, posterior lateral lateral suprasylvian area; PMLS, posterior medial lateral suprasylvian area; PS, posterior suprasylvian area; VA, ventral anterior nucleus; VLS, ventral lateral suprasylvian area; VM, vertical meridian. 
is the lateral posterior (LP)-pulvinar complex, and the focus of this report is the description of its relationship to the visual cortex.

In the cat the visual cortex extends beyond the boundaries of the striate area and even beyond the boundaries of the adjacent areas 18 and 19. In the early 1940's, Marshall and co-workers (1943) noted primary evoked responses in the classical "association cortex" of the middle suprasylvian gyrus. Later, evoked potential and single unit studies confirmed the existence of visual units in this "lateral suprasylvian" area (e.g., Clare and Bishop, 1954; Hubel and Wiesel, 1969). In a series of studies, Tusa, Palmer, and Rosenquist (Palmer et al., 1978; Tusa and Palmer, 1980; Tusa et al., 1978, 1979, 1981) identified a total of 13 topographic representations of the visual hemifield in the cat visual cortex. Some classically defined areas, such as areas 17,18 , and 19 , were each shown to contain a single representation of the visual hemifield, whereas the lateral suprasylvian area, for example, was shown to consist of six retinotopically defined areas. It is this parcellation of cortex, based upon retinotopic criteria, that is used in the present study.

The LP-pulvinar complex of the cat has been subdivided into at least three parallel zones on the basis of afferent input, architectonic differences, and histochemical activity (e.g., Rioch, 1929; Graybiel, 1972a, b; Niimi and Kuwahara, 1973; Updyke, 1977; Graybiel and Berson, 1980). Three of these zones have been mapped electrophysiologically, and each contains a single representation of the visual hemifield (Mason, 1978; Raczkowski and Rosenquist, 1981).

Previous anatomical studies have revealed important features of the connections of the LP-pulvinar complex with the visual cortex. Relying chiefly on anterograde tracing methods, these efforts have demonstrated that the main subdivisions of the LP-pulvinar complex are topographically and reciprocally connected with the extrastriate visual cortex (Graybiel, 1972b; Kawamura et al., 1974; Niimi et al., 1974; Berson and Graybiel, 1978). However, each of these studies was completed before the extensive cortical and thalamic maps were available and thus did not address essential questions regarding how each of the cortical and thalamic areas are interconnected. For example, does each thalamic subdivision have a unique set of cortical targets; and, do connectivity data support the cortical parcellation schema based upon retinotopy?

Some recent progress has been made in describing the connections of the cat's extrastriate visual areas. Anterograde studies in this laboratory (Symonds et al., 1981) suggest that each subdivision of the LP-pulvinar complex has a unique set of cortical targets and that thalamocortical connectivity patterns generally support the cortical parcellation based on retinotopy. However, the description of the cortical targets of these injections was based solely on reference to published mapping data. Other investigators (Hughes, 1980; Updyke, 1981) have used electrophysiological mapping in combination with anatomical tracing methods to investigate thalamocortical connectivity in the cat's extrageniculate visual pathways. However, these studies limited their focus to a few of the areas located in the lateral suprasylvian cortex.
The present report describes the patterns of thalamocortical interconnections of each of the 13 mapped visual cortical areas and adjacent regions for which no obvious retinotopy has yet emerged. We used electrophysiological mapping to identify each visual area prior to injection and, in doing so, confirmed previous mapping studies (Palmer et al., 1978; Tusa and Palmer, 1980; Tusa et al., $1978,1979,1981)$. The patterns of thalamic label identify new routes by which visual impulses gain access to the cortex, confirm previous functional parcellations of the LP-pulvinar complex, and identify other sources of thalamic inputs to the extrastriate visual cortex. Some of these findings have been reported previously (Raczkowski, 1979; Raczkowski and Rosenquist, 1980; Rosenquist et al., 1982).

\section{Materials and Methods}

The present experiments combined electrophysiological mapping studies of the visual cortex with the use of anterograde and retrograde anatomical tracers to determine thalamocortical interconnections. Single injections of horseradish peroxidase (HRP), alone or in combination with tritiated leucine, were made at cortical sites defined by microelectrode recording. Occasional exceptions to this strategy were made. These included cortical injections placed in nonvisuotopically organized or nonmapped areas such as the cingulate gyrus, parietal areas 5 and 7 , and insular fields. In addition, one stereotaxic injection of tritiated leucine was made into the posterior thalamus itself.

Thirty-three cats were prepared for electrophysiological recording following a protocol adopted from Tusa et al., 1978. We induced anesthesia with ketamine (20 mg/ $\mathrm{kg}$ ) and quickly inserted a venous cannula. The cat was then intubated and positioned in a stereotaxic head holder. (Supplemental doses of sodium thiopental were given intravenously, as needed, during subsequent procedures.) A bolt was cemented to the skull and secured to one arm of the stercotaxic instrument. This procedure fixed the head and allowed removal of the potentially painful eye, ear, and mouth restraining bars. In addition, all wound margins were infiltrated with a local anesthetic ( $2 \%$ mepivacaine hydrochloride). An opening was made in the skull overlying the visual cortex and the dura was reflected. This opening was covered with $3.5 \%$ agar in normal saline. Next, we paralyzed the cat with an injection of gallamine triethiodide administered through the venous cannula. Following paralysis, the animal was artificially respired, and expired end-tidal carbon dioxide was maintained at approximately $4.0 \%$. Anesthesia for the remainder of the experiment was provided by a nitrous oxide (75\%)/oxygen ( $25 \%$ ) mixture. A continuous intravenous infusion of gallamine triethiodide and $d$ tubocurarine suppressed residual eye movements during recording. Rectal temperature was kept at approximately 36 to $38^{\circ} \mathrm{C}$ and the heart rate was monitored. The pupils were dilated with $1 \%$ atropine sulfate and appropriate contact lenses were placed on the corneas. Using a reversible ophthalmoscope, retinal landmarks were plotted onto a 3 -ft. diameter hemisphere.

We recorded extracellular cortical activity with lac- 
quer-coated tungsten electrodes or saline-filled glass pipettes. Slits or spots of light were projected from a handheld projector onto the hemispheric screen, and receptive fields were plotted using the contralateral eye only. Electrode positions were plotted either on drawings of cortical blood vessels or by making small electrolytic lesions.

A sufficient number of cortical penetrations were made to allow us to identify at least one cortical area on the basis of its retinotopy in every experiment. After selecting a site for injection, we exchanged the recording electrode for a pipette (10- to $15-\mu \mathrm{m}$ tip) filled with $15 \%$ HRP and tritiated leucine (50 to $150 \mu \mathrm{Ci} / \mu \mathrm{l}$ ). During an injection penetration, we recorded multiple unit neural activity to verify the receptive field center of the injection site and then injected approximately $75 \mathrm{nl}$ of the HRP/ tritiated leucine mixture. Injection was by pressure introduced to the pipette holding chamber via a solenoidoperated valve.

Steps were then taken to reverse paralysis. We discontinued the infusion of paralytic drugs and closed the wound margins of the scalp and leg. The anesthetic gases were then discontinued and replaced with room air. Edrophonium chloride was injected intramuscularly, as needed, to speed the recovery from paralysis. When the cat was capable of breathing on its own, the endotracheal tube was removed and the cat was returned to its cage for 40 to $48 \mathrm{hr}$. Immediately after the reversal from paralysis and recovery from anesthesia, animals showed no signs of pain or discomfort. That they were capable of vocalization and withdrawal responses was confirmed by such responses to a pinch of the paw. Home cage postoperative behavior appeared normal.

At the time of sacrifice, the cat was deeply anesthetized with sodium pentobarbital $(40 \mathrm{mg} / \mathrm{kg})$ and perfused through the heart with saline followed by a mixture of $0.5 \%$ paraformaldehyde and $2.5 \%$ glutaraldehyde in 0.1 M phosphate buffer followed by $15 \%$ sucrose buffer. The brain was photographed, blocked, and immersed in cold sucrose buffer for $36 \mathrm{hr}$.

Coronal sections ( $48 \mu \mathrm{m}$ ) were cut on a freezing microtome and every sixth section was collected in saturated sodium sulfate and processed for HRP histochemistry using the chromogen o-dianisidine (de Olmos, 1977). All HRP-reacted sections were rapidly dehydrated and cleared in xylene, since counterstaining or excessive exposure to alcohol led to considerable fading of the reaction product. A second series was prepared for autoradiography (Cowan et al., 1972). Tissue was exposed for 8 to 16 weeks, developed, and stained with thionin. Another series, stained with hematoxylin, was also available for study.

Labeled cells and reduced silver grains were charted in outline drawings of relevant brain sections using a microscope equipped with a darkfield condenser and a drawing attachment. Next, cyto- and myeloarchitectonic features of each thalamus were studied and lines indicating changes in cell and fiber densities were added to the charts. Finally, each hemisphere was reconstructed, aided by photographs taken at the time of perfusion, and the locations of tracer uptake and electrode tracks were indicated on a drawing of the cortex.

\section{Results}

In the present study, we placed combined injections of HRP and tritiated leucine into physiologically identified loci within each of 13 retinotopically organized cortical visual areas. Injections were also made in several adjoining cortical areas which contain visually responsive neurons, but for which no obvious retinotopy has yet emerged. Charts depicting the distribution of label in the LP-pulvinar complex and other subcortical sites were made and related to the organization of the cortical mantle in terms of the area injected and its retinotopy. The location of each injection site and the spread of the heavy label from the site of injection were described in relation to the visuotopic maps of the cat cortex prepared by Tusa, Rosenquist, and Palmer (Palmer et al., 1978; Tusa and Palmer, 1980; Tusa et al., 1978, 1979, 1981). Label in the LP-pulvinar complex and other thalamic sites was located in relation to architectonic boundaries observed in individual experiments. The terminology of the LP-pulvinar complex followed the scheme developed below. The terminology of Guillery et al. (1980) was applied to the lateral geniculate complex. The terminologies of Jones and Powell (1971) and Morest (1964) were followed in describing the posterior thalamic nuclei, and those of Niimi and Kuwahara (1973) were used in describing the anterior and midline nuclei.

\section{Subdivisions of the lateral posterior-pulvinar complex}

In order to describe meaningfully the topographic pattern of thalamic connections with the visual cortex, it is necessary to understand the organization of the LPpulvinar complex. Attempts to distinguish functional subdivisions within this region based on cytoarchitectonic criteria alone have led to a number of conflicting schemes (Rioch, 1929; Niimi and Kuwahara, 1973). Consequently, efforts have been made to subdivide this region on the basis of its connections. Two principal schemes have been advanced to date, and in both four subdivisions of the extrageniculate visual thalamus are recognized. In the first, three subdivisions of the LPpulvinar complex, pulvinar nucleus, and the lateral (LPl) and medial (LPm) divisions of the lateral posterior nucleus, were identified on the basis of receiving projections from the pretectum, striate cortex, and superficial layers of the superior colliculus, respectively. A fourth subdivision, Rioch's "posterior nucleus," was identified on the basis of its cytoarchitectural quality and its connections with striate cortex (Rioch, 1929; Graybiel, 1972a, b). In the second scheme, Rioch's "posterior nucleus" was incorporated with the rest of the striatedrecipient zone into one nuclear complex termed LPI. In addition, the domain of the extrageniculate visual thalamus was expanded medially to allow for a new subdivision (Updyke, 1977). In this latter arrangement, the designation for the tectorecipient zone was changed from LPm (of Graybiel, 1972a, b) to LPi and the term LPm was reserved for the new zone lying medial and rostral to the tectorecipient zone.

We have addressed the question of the functional subdivision of the extrageniculate visual thalamus by 

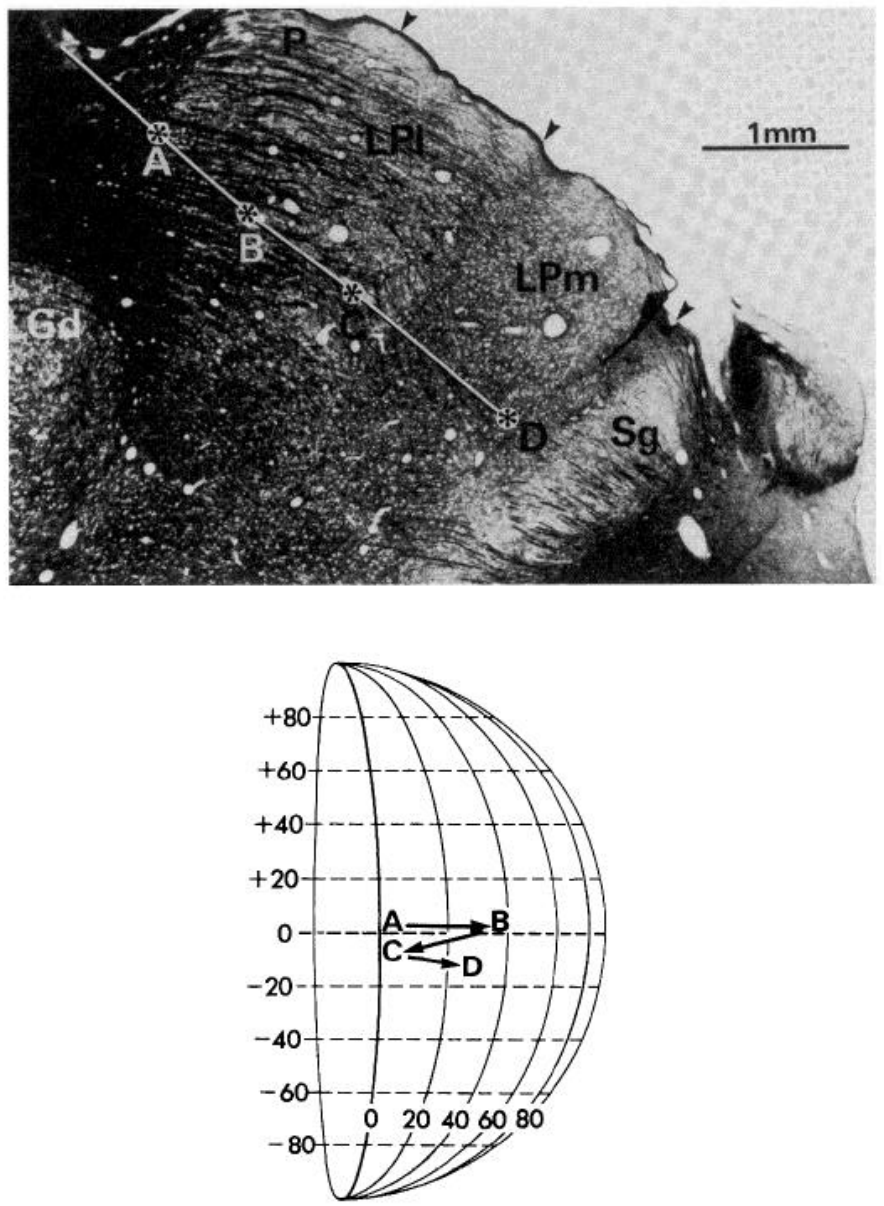

Figure $1 .^{5}$ Top, Photomicrograph of a myelin-stained coronal section through the LP-pulvinar complex showing a single reconstructed electrode penetration. Points $A, B, C$, and $D$, where reversals in the progress of visual receptive fields were seen, are marked by lesions. Arrowheads indicate changes in myeloarchitectonic quality. Bottom, Perimeter chart of visual hemifield indicating the progression of receptive field centers encountered by the recording electrode from point $A$ to point $D$ (top). For further details see Raczkowski and Rosenquist (1981).

using still another criterion, namely, that of retinotopy (Raczkowski and Rosenquist, 1981). We have mapped visually driven units in the posterior thalamus and identified three separate representations of the visual field by associating receptive field centers with recording points along each electrode penetration. Figure 1 illustrates one electrode penetration from a mapping experi-

\footnotetext{
${ }^{5}$ The abbreviations used on all figures are: $A D$, anterior dorsal nucleus; $A M$, anterior medial nucleus; $A V$, anterior ventral nucleus; $C G$, cingulate gyrus; $C e M$, centre median nucleus; $H a$, habenula nucleus; $L G v$, ventral lateral geniculate nucleus; $M D$, medial dorsal nucleus; $M G d$, dorsal division of medial geniculate nucleus; $M G m$, medial division of medial geniculate nucleus; $M G v$, ventral division of medial geniculate nucleus; $P, P u l$, pulvinar; $P f$, parafascicular nucleus; $P O, P o$, posterior group of thalamic nuclei; $R$, reticular nucleus; $R h$, rhomboid nucleus; $S g$, suprageniculate nucleus; $S V A$, splenial visual area; $V L$, ventral lateral nucleus; $V M$, ventral medial nucleus; $V P$, ventral posterior nucleus ( $\mathrm{VPl}$ and $\mathrm{VPm}$ ); $\mathrm{VPl}$, ventral posterior lateral nucleus; $V P m$, ventral posterior medial nucleus.
}

ment in which evidence is seen for one representation of the visual hemifield in the pulvinar and for two representations in the LP. Cells medial to point $D$ in Figure 1 could not be visually driven. For a more detailed analysis of these mapping experiments refer to Raczkowski and Rosenquist (1981). On the basis of rules of retinotopic representation, we were unable to find any separable entity corresponding to the "posterior nucleus" of Rioch (1929). Thus, we have incorporated it into the striate-recipient zone and refer to the entire zone as LPl (see Updyke, 1977). However, in light of its obvious cytoarchitectonic quality, we have often indicated the boundaries of Rioch's nucleus in our drawings.

Since we found the region of the posterior thalamus designated as "LPm" by Updyke (1977) to be visually unresponsive (Raczkowski and Rosenquist, 1981), we elected not to include it in our scheme of the extrageniculate visual thalamus. This sector is not connected with any of the contiguous areas of the visual cortex, but rather with a circumscribed region within the sylvian gyrus (Fig. 2). Following an injection of tritiated leucine into this medial thalamic zone, labeled fibers were followed laterally through the LP-pulvinar complex on their way to the lateral thalamic peduncle. Upon reaching the peduncle, these fibers curved rostrally and terminated principally in layers I and IV of the ventral bank and crown of the sylvian cortex where auditory evoked potentials have been recorded (Woolsey, 1961). Neither the superficial layers of the superior colliculus or the pretectum, nor the striate cortex innervate the thalamic zone sending axons into this cortex. Also, this cortical area is not a target of the parvocellular $\mathrm{C}$ laminae of the dorsal lateral geniculate nucleus, which project widely upon other mappable visual areas (Raczkowski and Rosenquist, 1980).

On the basis of recent findings, other subdivisions of the LP-pulvinar complex have been proposed. Intercalated between the medial interlaminar nucleus (MIN) and the lateral edge of the LP-pulvinar complex is a thin marginal strip which receives direct input from the retina. This marginal zone has been considered either a new subdivision of the pulvinar nucleus (e.g., Berman and Jones, 1977; Leventhal et al., 1980) or an extension of the parvocellular C laminae (Guillery et al., 1980). Our data favor the latter conclusion. The cortical targets of this marginal zone closely resemble the cortical projections of the parvocellular C laminae (see Fig. 3). Furthermore, this marginal zone receives input from very small (presumably W) ganglion cells (Kawamura et al., 1979), a feature also shared by the parvocellular C laminae (Itoh et al., 1981).

Two additional subdivisions of the LP-pulvinar complex, identified using connectional criteria, have been proposed. The first is a thin dorsal extension of the "geniculate wing" which receives input from the deep cerebellar nuclei (Itoh et al., 1979). The second is a thin strip of cells located along the medial edge of the pulvinar nucleus, at rostral levels, which receives input from the hypothalamus (Fujii and Yoshii, 1979). This zone is also connected with the cingulate cortex (Fig. 27). However, in our mapping studies (Raczkowski and Rosenquist, 


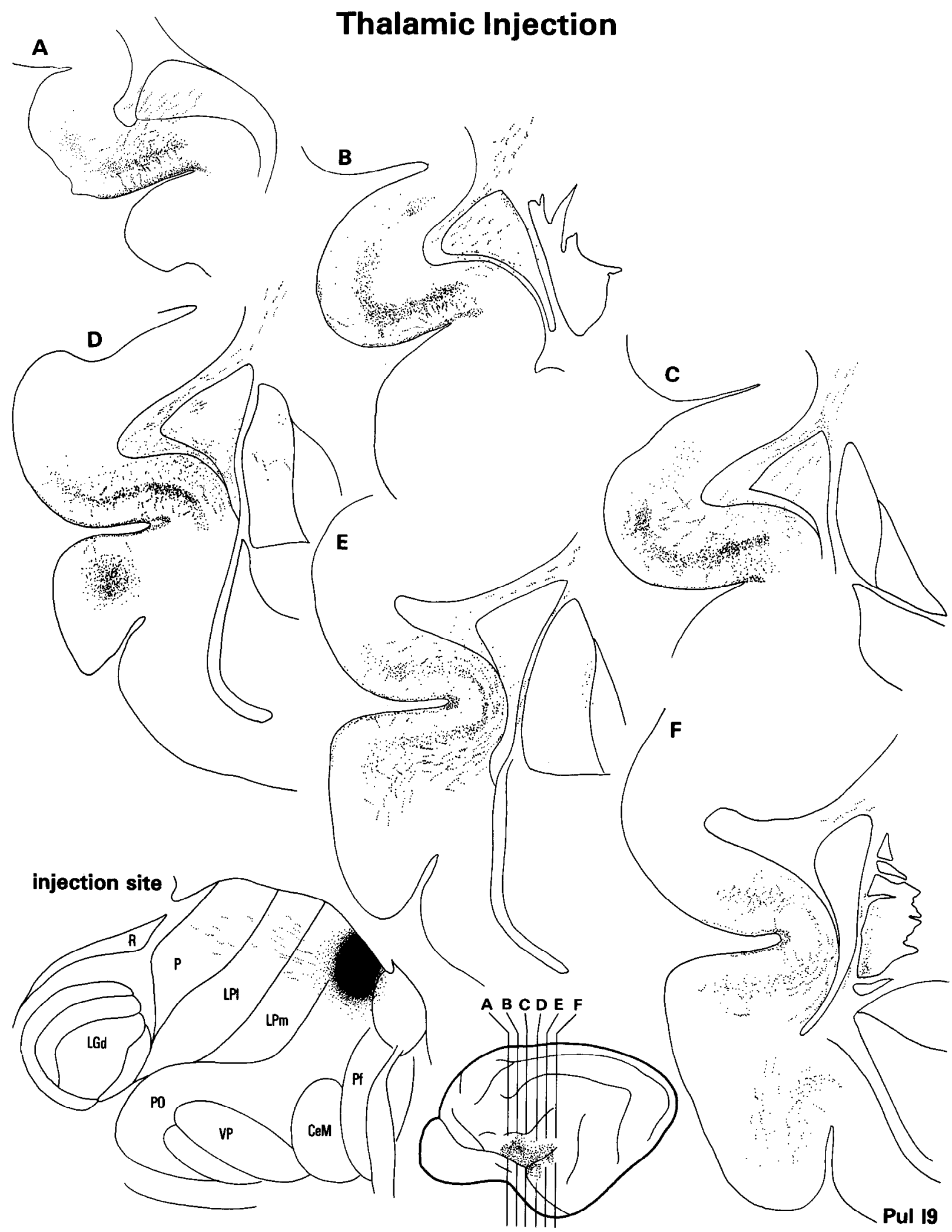

Figure 2. Charts of silver grains (black dots) in coronal sections ( $A$ to $F$ ) of the cat's sylvian gyrus after injection of tritiated leucine in the thalamic zone medial to the LPm. A lateral view of the cat brain indicates the level of each section $(A$ to $F)$. The injection site is indicated on a coronal view of the cat's thalamus.

1981), we were unable to uncover any retinotopic identity corresponding to either of these two projection zones.

In summary, our retinotopic mapping experiments suggest three functional subdivisions of the extrageniculate visual thalamus. In the anatomical connectivity experiments which follow, we did not independently confirm the borders of the three subdivisions of the LP. pulvinar complex by retinotopic mapping. Thus, the issue arose of how best to identify and depict these borders in our charts of thalamic label. For this, we relied on two 


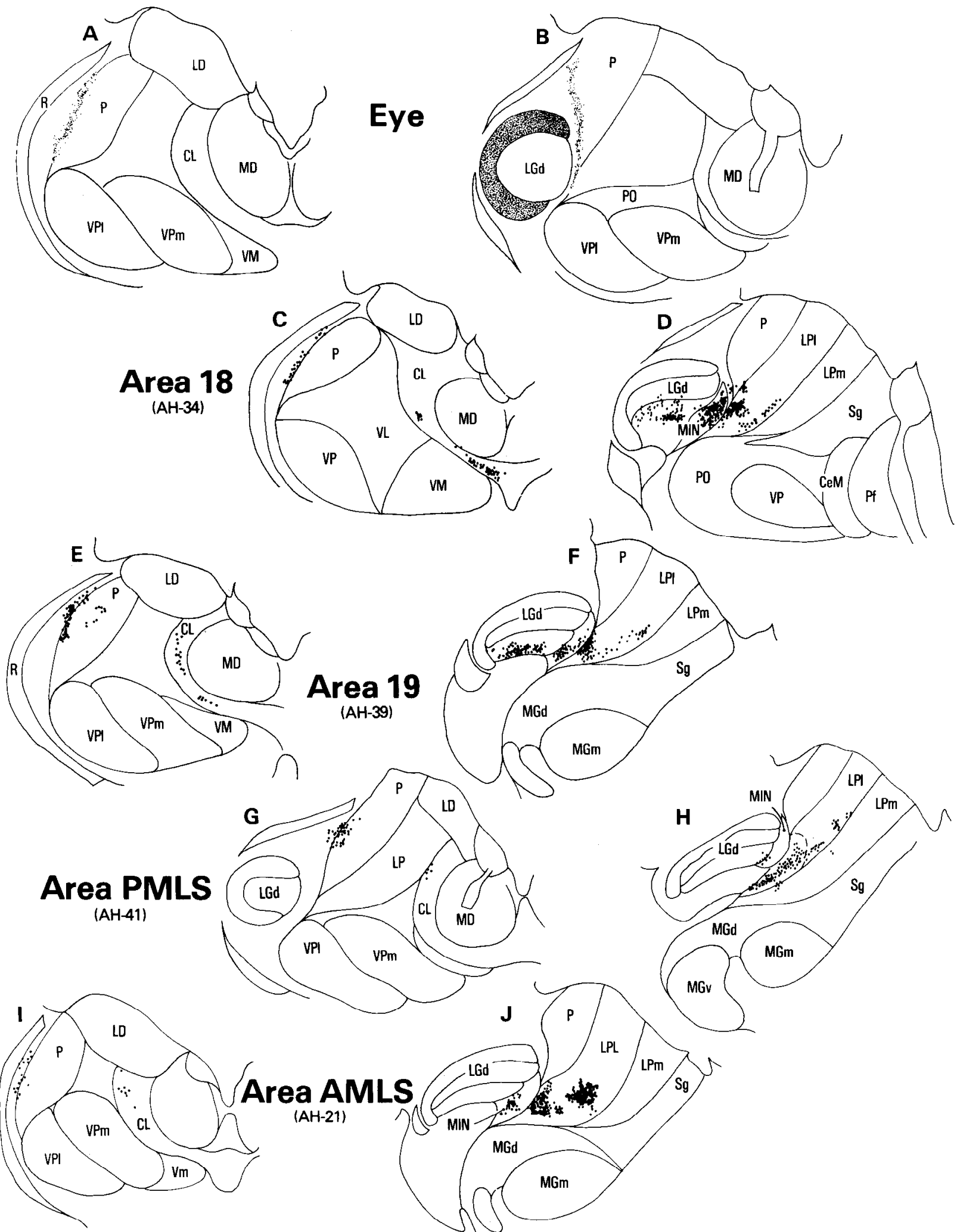

Figure 3. Connections of the "geniculate wing" of the LGd. $A$ and $B$ depict the location of silver grains (black dots) following injection of tritiated leucine into the contralateral eye. The "geniculate wing" appears as a narrow strip of label along the lateral margin of the pulvinar $(P) . C$ to $J$ show four pairs of thalamic coronal sections depicting HRP-labeled neurons in the "geniculate wing" and nearby thalamic regions after different cortical injections: $C$ and $D$, area $18 ; E$ and $F$, area $19 ; G$ and $H$, area PMLS; $l$ and $J$, area AMLS.

separate criteria, each related to retinotopy. First, as can be seen in Figure 1, myeloarchitectural differences among the three subdivisions were sometimes apparent and could be used to help guide our identification of borders.
Second, our mapping study of the LP-pulvinar complex, together with the connectivity study of Updyke (1977) resulted in "standard maps" to which our anatomical sections could be related. 


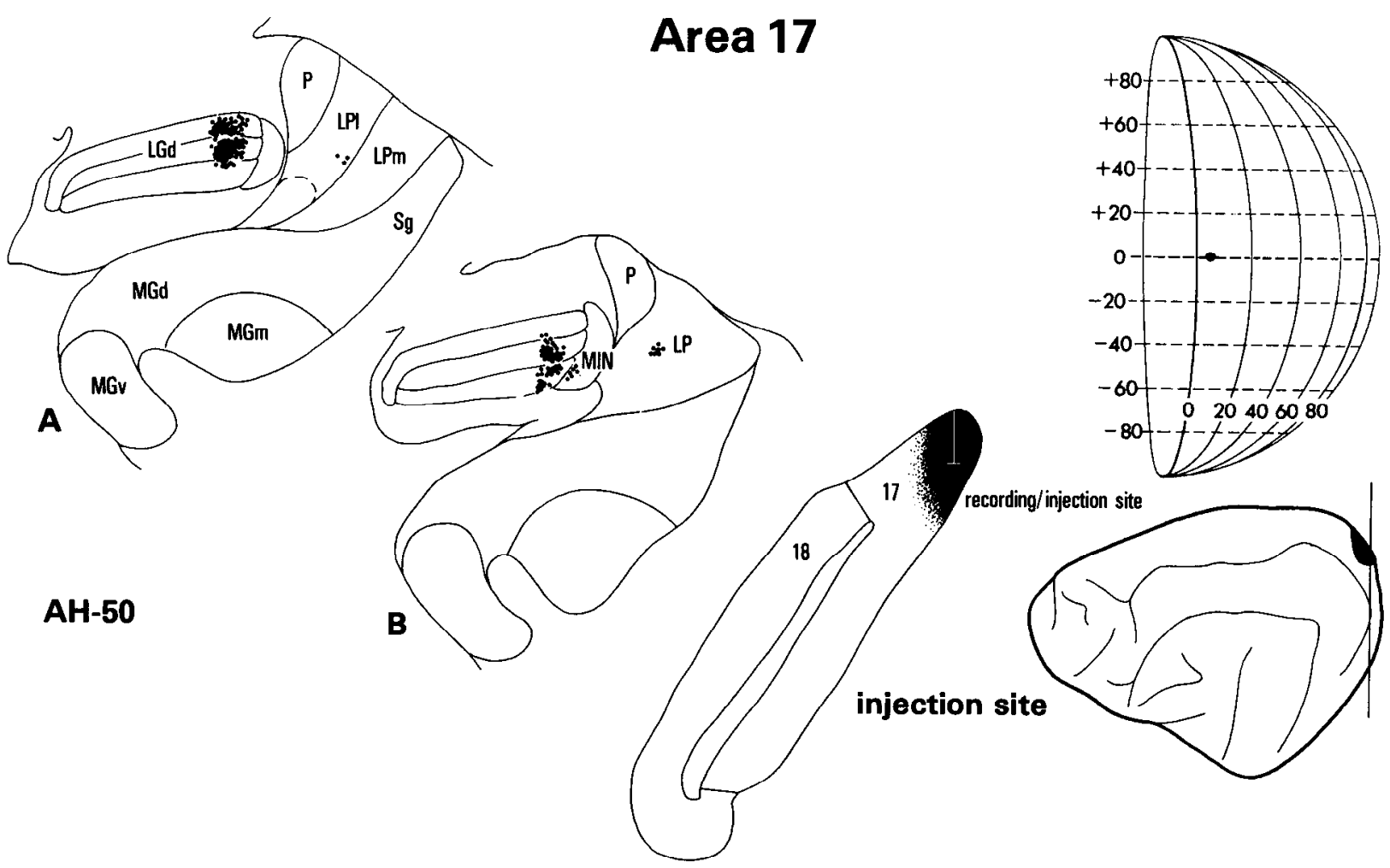

Figure 4. Chart of HRP-labeled cells in the visual thalamus $(A$ and $B)$ after injection of HRP into area 17. The receptive firld of the injection site in area 17 is depicted on the perimetry chart.

\section{General features of LP-pulvinar complex connections with the visual cortex}

Several features regarding the connections between the LP-pulvinar complex and the visual cortex are useful to summarize before describing the specific findings. First, our combined HRP and tritiated leucine injections indicated complete reciprocity in thalamocortical and corticothalamic projection systems, and as a result, the two experimental strategies can be employed independently to describe thalamocortical interconnections. Second, the pattern of label in the LP-pulvinar complex generally formed slabs elongated in the dorsoventral dimension, when viewed in cross-section. Third, comparable injections in similar retinotopic loci in two areas often produced slabs of label of different thickness or extent. Fourth, in addition to reciprocal, slab-like distributions of label in the LP-pulvinar complex, we have also seen reciprocal label in the parvocellular $\mathrm{C}$ laminae of the LGd after injections into nearly every extrastriate visual cortical area. Two other thalamic nuclei, the ventral anterior (VA) and central lateral (CL), which are not usually considered as part of the visual path, consistently contained label following many of these injections. Reciprocal claustrocortical interconnections and descending cortical projections to the superior colliculus, pretectum, pons, and other brainstem centers also were observed and are the subjects of other reports.

Because we wanted to examine the relationship between connections and representations of the visual field in both the thalamus and the cortex, three standards were established for selecting the experiments to be illustrated below. First, it was necessary to illustrate label in all subdivisions of the LP-pulvinar complex and to do so required illustrating injections from several different cortical areas. Second, the injections had to be in different retinotopic locations so that if any anatomical evidence for a topographic map of the posterior thalamus existed, it would emerge. Third, we chose at least one example from each cortical visual area so as to understand its individual pattern of thalamic connections.

\section{Central vision and the vertical meridian}

The representation of central vision in the posterior thalamus is illustrated in four experiments (Figs. 4, 5, 8, and 9). The first experiment in this set shows an injection of HRP placed in area 17 at a retinotopic locus $5^{\circ}$ removed from the vertical meridian (VM) and straddling the horizontal meridian (HM) (Fig. 4). The retinotopic locus of our injection site was confirmed in the LGd complex where most of the labeled cells were seen in the A layers, with a few cells in the $C$ laminae and the adjacent MIN. In the extrageniculate thalamus, a small focus of label was observed in the caudal one-third of the LP-pulvinar complex. The label bisected the LP and was located just lateral to a change in the density of fibers coursing through the complex.

In order to show the location of central vision in other zones of the LP-pulvinar complex, which are not connected with area 17 , we must illustrate cases in which extrastriate areas were injected. A small combined HRPleucine injection was placed in the region of the border between the posterior lateral (PLLS) and dorsal (DLS) lateral suprasylvian areas at a retinotopic locus nearly identical to that of the previous experiment (Fig. 5). The 


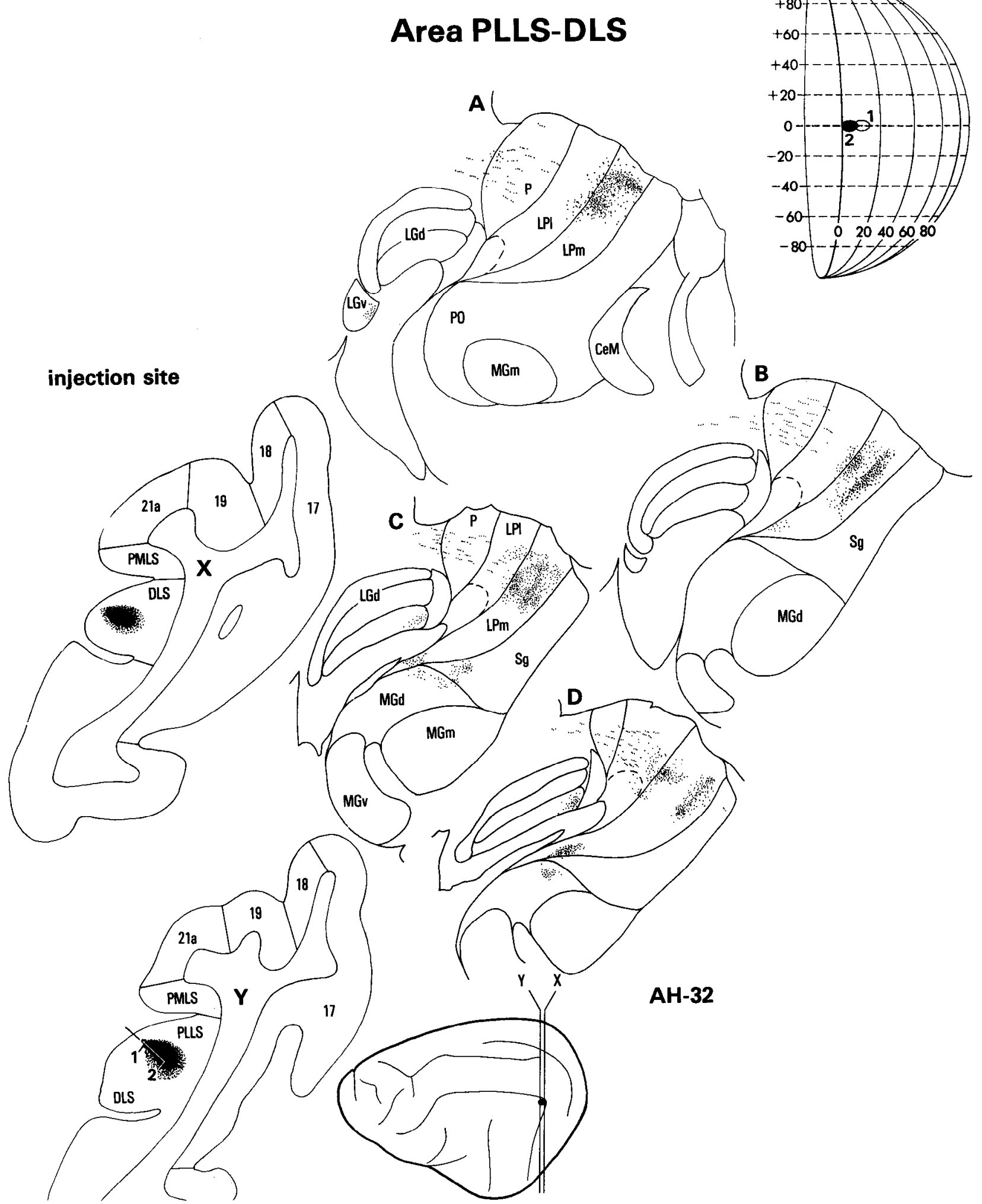

Figure 5. Charts of silver grains (black dots) in coronal sections ( $A$ to $D$ ) of the cat's thalamus after an injection of tritiated leucine into areas PLLS and DLS. The cortical injection site is indicated in coronal sections $X$ and $Y$ and on the dorsolateral view of the brain. The receptive fields recorded at points 1 and 2 (section $Y$ ) are illustrated in the perimeter chart. The injection was made at point 2 .

pattern of HRP and autoradiographic label overlapped extensively in the LGd and LP-pulvinar complex. We have depicted the orthograde transport because it re- flected the pattern of thalamic label more dramatically. The retinotopy of the injection site was confirmed in the projections to the parvocellular C laminae of the LGd. 
(A photomicrograph of this label is seen in Fig. 6). The predominate focus of label in the LP-pulvinar complex was in its LPm subdivision along its border with the LPl. A small focus of label may also be present in the LPl, although this could also be due to a small error in our demarcation of the LPl-LPm border. An important point to note is that although this injection was rather restricted in size and retinotopic locus, the label in the $\mathrm{LPm}$ was quite extensive in both the anterior-posterior and medial-lateral dimensions. Furthermore, in this densely labeled sector of the LPm, the grain pattern was not uniform, but rather interrupted at irregular intervals by sectors that were sparsely labeled (see Fig. 7).

Figure 8 illustrates the HRP label from an experiment with an injection into area $21 \mathrm{a}$ at a retinotopic locus centered $8^{\circ}$ from the VM and straddling the HM. HRPlabeled cells were seen in all three subdivisions of the LP-pulvinar complex. The heaviest concentration of labeled cells was located in the LPl along its common border with the LPm representing the VM. The heavy label in the LPl expanded laterally but failed to reach the lateral edge of the nucleus representing the visual periphery. At caudal levels in the posterior thalamus, some label extended medially into the LPm. Rostrally, a few retrogradely filled neurons were observed near the lateral edge of the thalamus in the pulvinar nucleus. Finally, a small patch of label ventral to the main band in the $\mathrm{LPl}$ was observed.

One final experiment illustrates the representation of the VM in the thalamus. The injection was located in the posterior medial lateral suprasylvian area (PMLS) at its border with the anterior medial lateral suprasylvian area (AMLS) at a retinotopic locus on the VM, some $22^{\circ}$ below the HM (Fig. 9). Confirming the location of the injection, labeled cells were seen on either side of the common border shared by the parvocellular $\mathrm{C}$ layers and the MIN. This label is illustrated in the photomicrograph in Figure 10. In the LP-pulvinar complex, most of the HRP (illustrated) and autoradiographic label was again located predominantly in the medial half of the LPl, along its common border with the LPm representing the VM. Observe that the heavy label in the LPI remained ventral at all anterior-posterior levels and extended

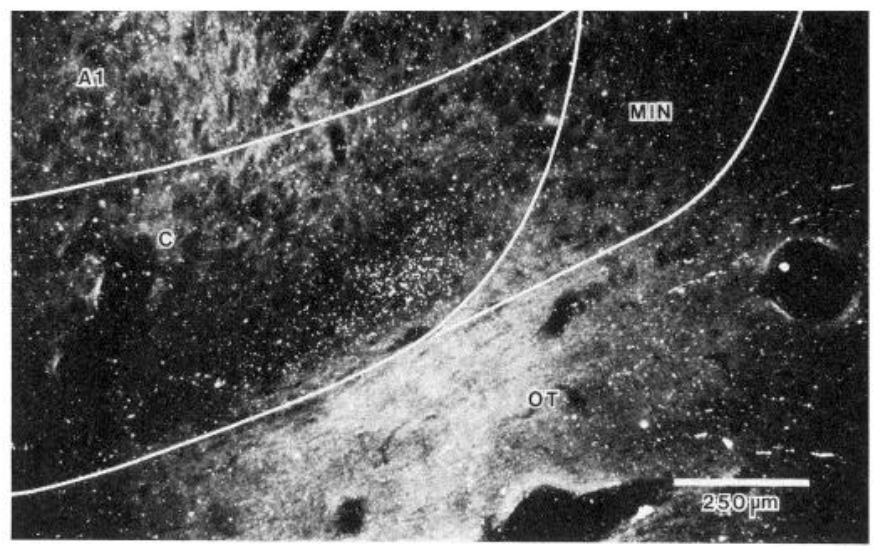

Figure 6. Darkfield photomicrograph showing silver grains in the parvocellular C laminae of the LGd following the injection of tritiated leucine illustrated in Figure 5.

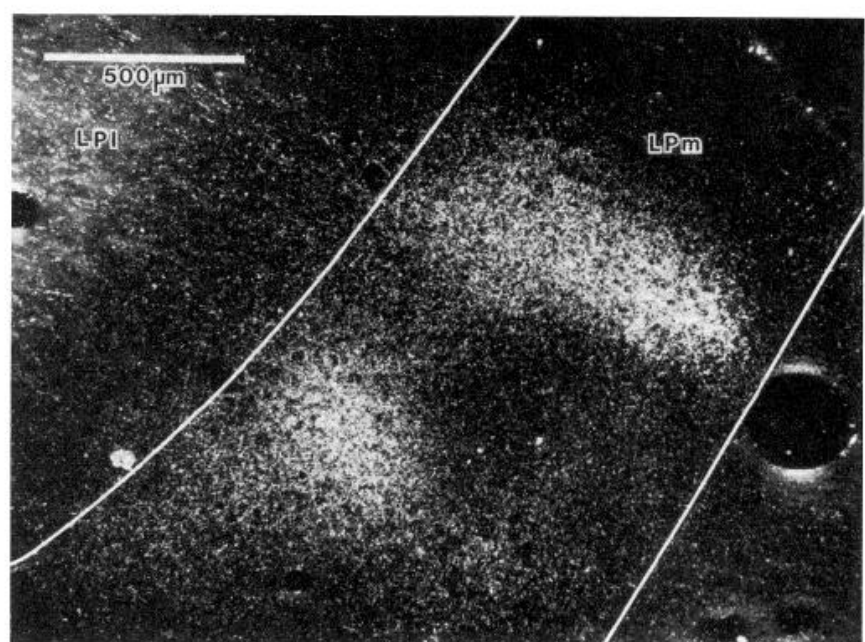

Figure 7. Darkfield photomicrograph showing silver grains in the LPm following the injection of tritiated leucine illustrated in Figure 5.

across the border of Rioch's posterior nucleus. This indicates that lower visual fields are located ventrally in the LP-pulvinar complex and that the architectonic salience of Rioch's nucleus is not reflected by a special set of connections or retinotopic unity. A small amount of label was also seen along the extreme lateral edge of the pulvinar nucleus at rostral thalamic levels. This zone seems to correspond to the zone which receives direct retinal input (see Fig. 3). Furthermore, previous work in our laboratory (Symonds et al., 1981) failed to reveal label in area PMLS after injections of tritiated leucine into the main body of the pulvinar proper. For these reasons, we feel that the HRP label observed along the lateral margin of the extrageniculate visual thalamus is best interpreted as lying within the borders of the geniculate complex.

In summary, our anatomical connectivity data support the notion that the VM is represented along the LPlLPm border and again along the lateral margin of the pulvinar nucleus. The injections illustrated in Figures 5 and 8 labeled large regions of both subdivisions of the LP despite being restricted to the representation of a small area of the visual field near the VM. This confirms our observation from mapping experiments and may reflect the fact that many receptive fields in the LPpulvinar complex include the VM despite being centered some distance away (Raczkowski and Rosenquist, 1981).

\section{Upper visual fields}

The next four experiments illustrate the pattern of thalamic label following injections into upper visual field representations of several additional extrastriate visual areas. Because the representation of upper fields is not extensive in many extrastriate areas, many of the "upper" fields illustrated lie relatively near or include the HM.

In Figure 11, we show an experiment in which an injection was made into the ventral lateral suprasylvian area (VLS), located on the ventral bank of the posterior suprasylvian sulcus. The receptive field center of the injection site was located $22^{\circ}$ away from the VM and $10^{\circ}$ 


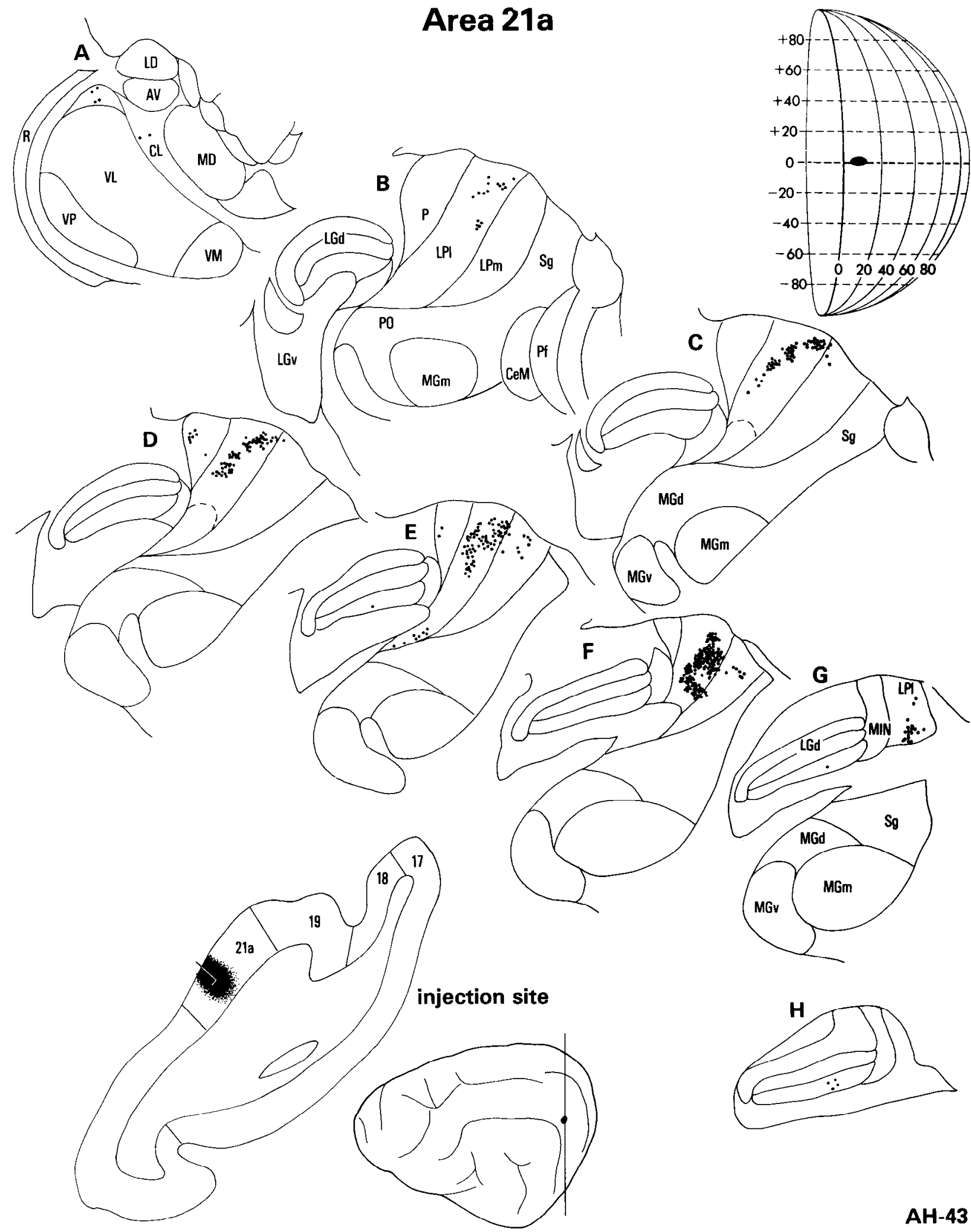

Figure 8. Chart of HRP-labeled cells in the visual thalamus ( $A$ to $H$ ) following injection of HRP into area 21a. The electrode track is illustrated on the coronal section of the cortex. The receptive field is illustrated on the perimetry chart.

above the HM. The location of HRP-labeled cells in the caudal parvocellular $\mathrm{C}$ laminae of the LGd confirmed the retinotopy of the injection site. HRP (illustrated) and autoradiographic label were seen in both subdivisions of the LP but were heaviest in the medial subdivision. Label remained dorsal and caudal except for a small discontinuous patch located far ventrally in the LP. As in most other extrastriate injections, HRP-labeled cells were also seen in the CL.

In the second experiment (Fig. 12), an injection was 

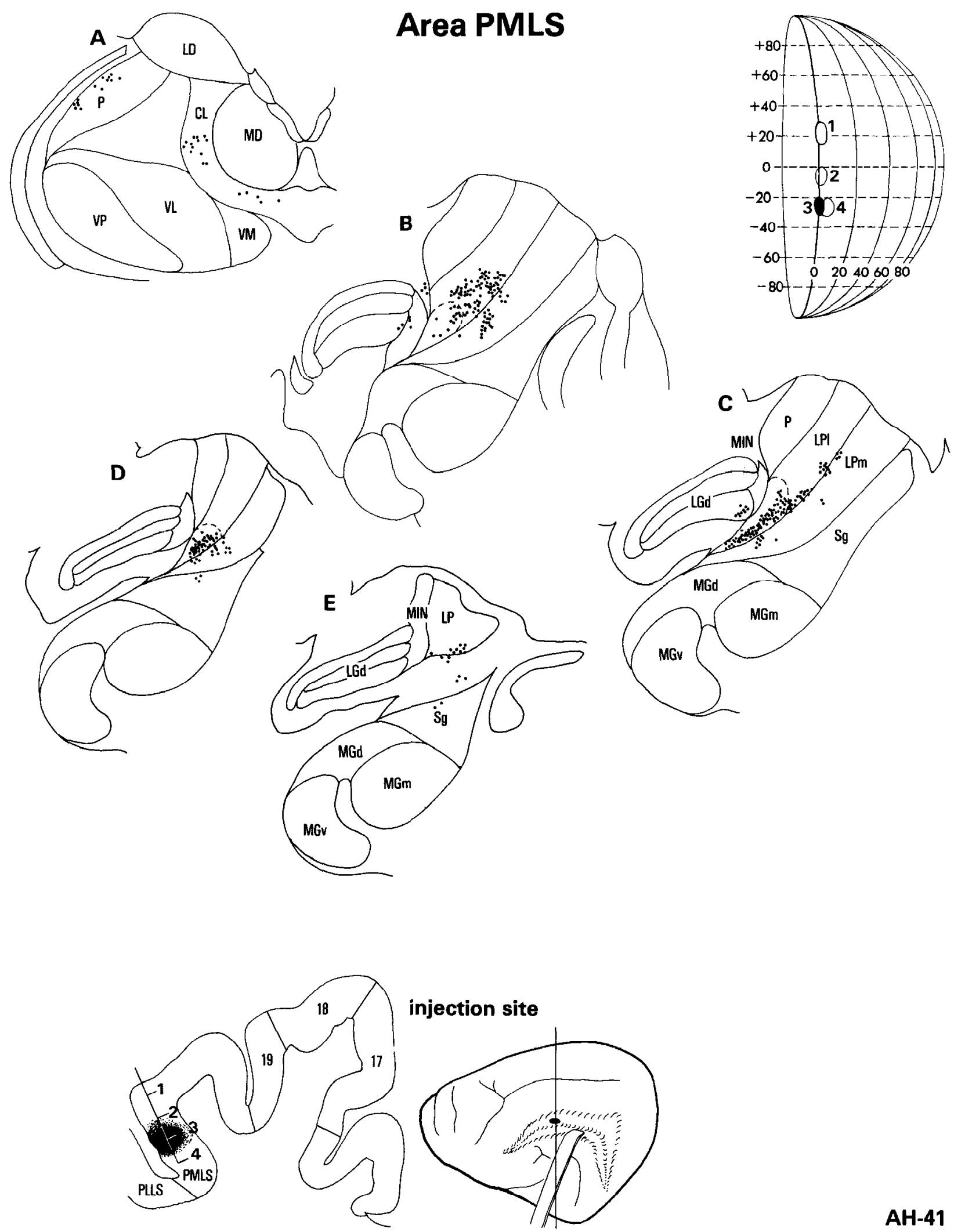

$\mathrm{AH}-41$

Figure 9. Chart depicting HRP-labeled cells in the visual thalamus after an injection of HRP into cortical area PMLS. The electrode penetration and recording sites ( 1 to 4 ) are illustrated in the coronal section of the cortex. Receptive field loci for each site are depicted and numbered on the perimetry chart. The injection was made at point 3.

placed in area $21 \mathrm{~b}$ at approximately the same retinotopic locus recorded in the previous experiment. 'The heaviest concentration of label again remained dorsally in the LPpulvinar complex. Caudally, a dense but patchy focus of label was located in the LP where we were unable to distinguish medial and lateral subdivisions. At intermediate levels, HRP labeling was most prominent in the $\mathrm{LPl}$, but the adjacent LPm and pulvinar subdivisions 


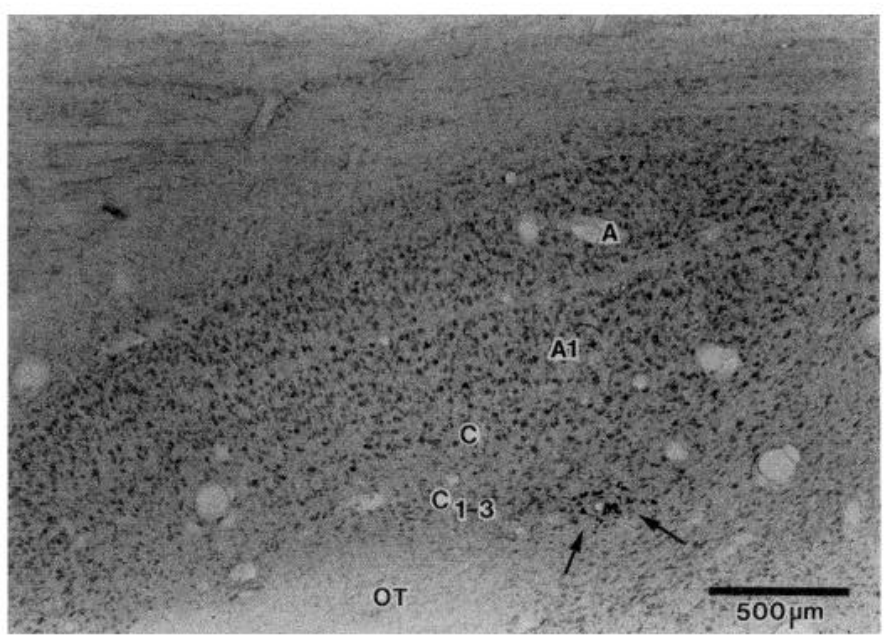

Figure 10. Brightfield photomicrograph of the LGd showing HRP-labeled cells (arrows) in the parvocellular C layers $\left(C_{1-3}\right)$ after the injection in Figure 9. $A, A 1, C$, and $C_{1-3}$ are respective geniculate laminae.

also contained a modest number of labeled neurons. Thalamic labeling in the LPl continued into the rostral aspect of the LP-pulvinar complex. No orthograde or retrograde label was observed in the LGd complex.

In the third experiment (Fig. 13), an injection of HRP was made into area 19 at a retinotopic locus similar to that recorded in the two previous experiments. (See Fig. 14 for a photomicrograph of this injection site.) The retinotopic locus of the injection site was confirmed by the pattern of HRP-labeled cells in the geniculate complex. In the laminated part, a column of label was seen laterally and caudally, extending through all layers of the $\mathrm{C}$ complex. In the MIN, label was distributed along its medial border. In the extrageniculate thalamus, the LPl contained the greatest number of labeled neurons. With the exception of the caudal pole, all of the labeled cells were situated dorsally. This label extended as a column into the rostral aspect of the nucleus. A lesser number of HRP-filled neurons were present in the adjacent LPm and pulvinar nuclei. Finally, a number of labeled cells were observed in both the CL and central medial (CM) nucleus.

The fourth cortical injection (Fig. 15) was placed in area $20 \mathrm{~b}$ which lies on the medial wall of the hemisphere. Because of its close proximity to areas 17 and 20a, a number of microelectrode penetrations were made in the same vicinity to ensure, using retinotopic criteria, that the injection was indeed localized to area 20b. Although the distribution of thalamic HRP and tritiated leucine label overlapped closely, we have illustrated the tritium because the limits of the autoradiographic injection could be accessed more confidently (see Fig. 16). In the LGd complex labeled cells and terminals were seen both in the parvocellular C laminae and in the MIN, again at retinotopic loci consistent with that recorded at the injection site. In the extrageniculate thalamus, three distinct foci of label were evident, one each in the LPm, $\mathrm{LPl}$, and pulvinar subdivisions. Note that the zones of label were restricted to the dorsal aspects of each subdivision. Furthermore, the labeling in both subdivisions of the LP extended farther caudally and rostrally than the label in the pulvinar nucleus. Although not apparent in the illustrated autoradiographic material, study of the HRP-reacted tissue from this experiment also revealed a projection from the CL, CM, and VA.

To summarize, the four previous experiments show that the upper visual field representation is situated dorsally and caudally in each of the three subdivisions of the LP-pulvinar complex.

\section{Lower visual fields}

Three experiments were selected to show the location of the lower field representation. Two of these injections involved areas on either bank of the middle suprasylvian sulcus. In the first experiment, the injection was placed in the anterior lateral lateral suprasylvian area (ALLS) at a retinotopic locus centered $30^{\circ}$ away from the VM and $15^{\circ}$ below the HM (Fig. 17). Although the injection was quite restricted in size, the pattern of autoradiographic (illustrated) and HRP thalamic label was complex. In LP-pulvinar, the densest label was displaced rostrally and ventrally over the LPm. This is consistent with mapping evidence that the rostral part of the LPm contains a representation of the lower quadrant of the visual field (Raczkowski and Rosenquist, 1981). The terminal label was distributed over the LPm in an irregular, patchy pattern. A very light patch of label was seen at one level in the pulvinar along its border with the LPl. A significant amount of label was also present over the medial geniculate complex and the posterior group. The label was heaviest over the dorsolateral portion of the suprageniculate nucleus where it adjoins the LPm. Sparse label was observed over the dorsal and medial subdivisions of the medial geniculate body. Since it is difficult to determine the boundary of this visual area with the periauditory cortex, it is not clear how much of this terminal label in the medial geniculate nucleus is attributable to injecting area ALLS. Rostral to the LPpulvinar complex, HRP and autoradiographic label was observed in the CL. Retrogradely filled HRP neurons also were seen in the adjacent CM. No label was seen in either the LGd or the MIN.

Another injection was placed into area AMLS, which lies on the medial bank of the middle suprasylvian sulcus just opposite area ALLS. The retinotopic locus of the injection within area AMLS was centered $35^{\circ}$ away from the VM and $35^{\circ}$ below the HM (Fig. 18). In the thalamus, no HRP (illustrated) or autoradiographic label was seen in the LGd, but the position of label ventrally in the MIN served to confirm the retinotopic locus of injection. In the LP-pulvinar complex, the vast majority of HRPlabeled cells were situated ventrally and rostrally in the LPl. The retrogradely filled neurons were distributed in the LPl in an irregular manner, reminiscent of the distribution of labeled terminals over the LPm following an injection into area ALLS. Behind the caudal limit of the labeling in the LPl, some HRP-filled neurons were observed in the LPm, along its border with the suprageniculate nucleus. Additional label was seen along the far lateral edge of the pulvinar complex. Since this region of the pulvinar complex contains a representation of central vision, and this injection was situated in a representation of the peripheral visual field, we feel this label should be 


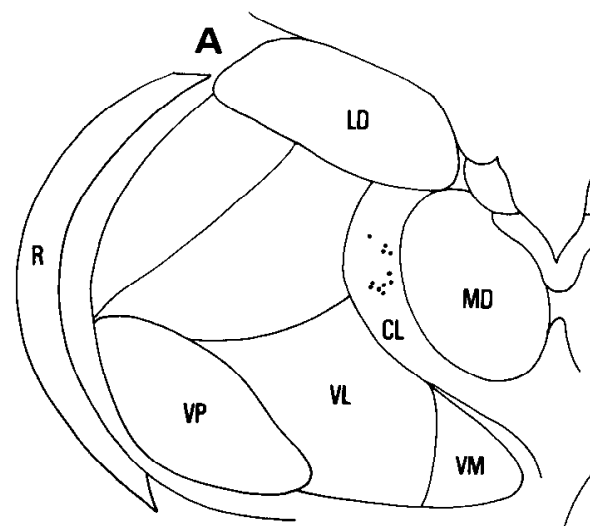

Area VLS

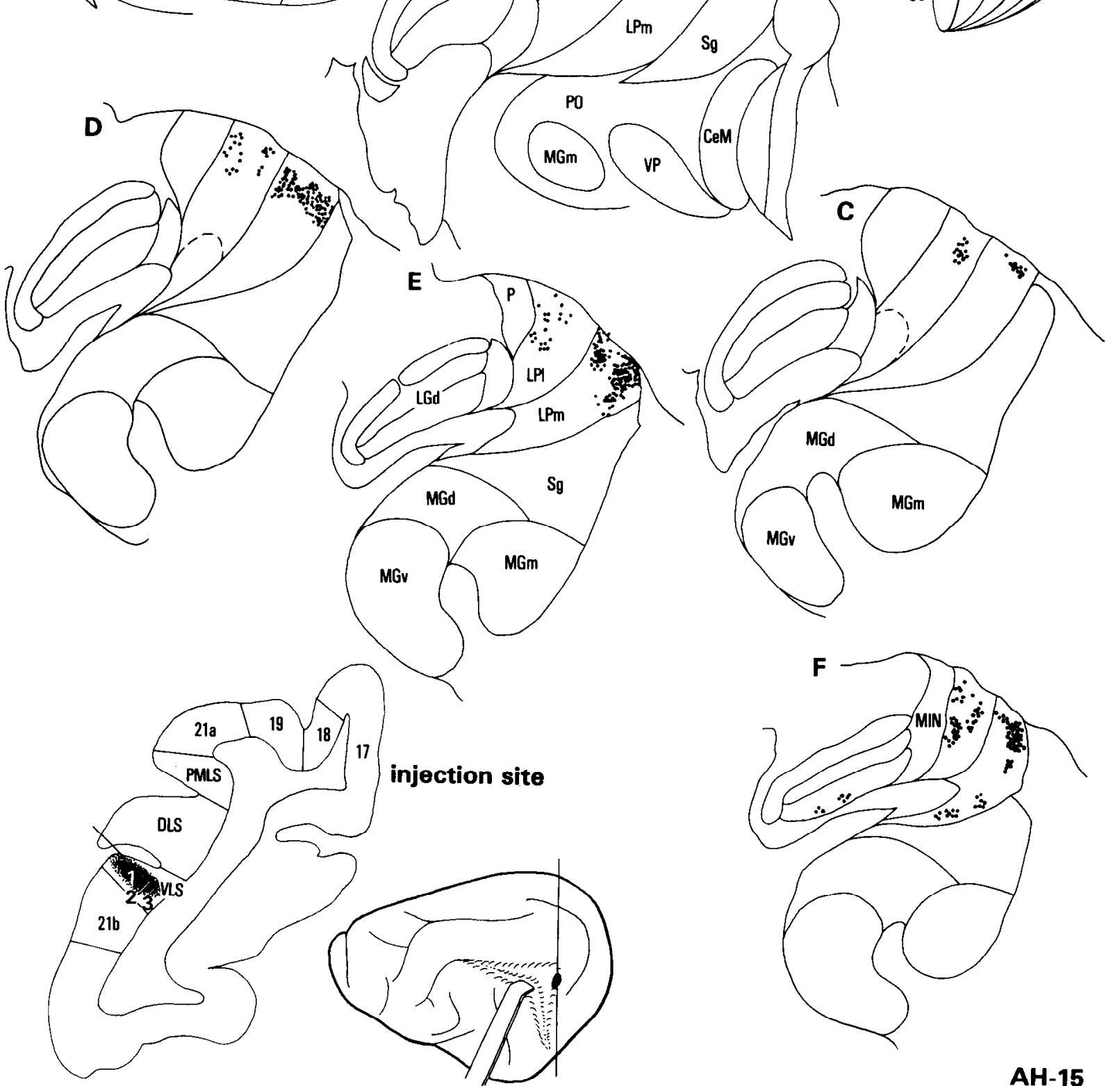

Figure 11. Chart of HRP-labeled cells in the cat's visual thalamus ( $A$ to $F$ ) after an HRP injection into cortical area VLS. The electrode track recording sites ( 1 to 3 ) and injection site are seen in the coronal section of the cortex. The receptive fields recorded at each of the recording sites are illustrated on the perimeter chart. The injection was made at point 2.

ascribed to the "geniculate wing" of the lateral geniculate complex. HRP-labeled cells were also seen in the CL and VA.

In a third experiment (Fig. 19), an injection was made into area 18 at a retinotopic locus comparable to that recorded in the previous experiment. The retinotopy of the injection site was confirmed by the location of HRPlabeled cells in the rostral LGd and MIN. Figure 20 illustrates some of the label in the A layers of the LGd. A portion of the retrograde label in these nuclei and the 


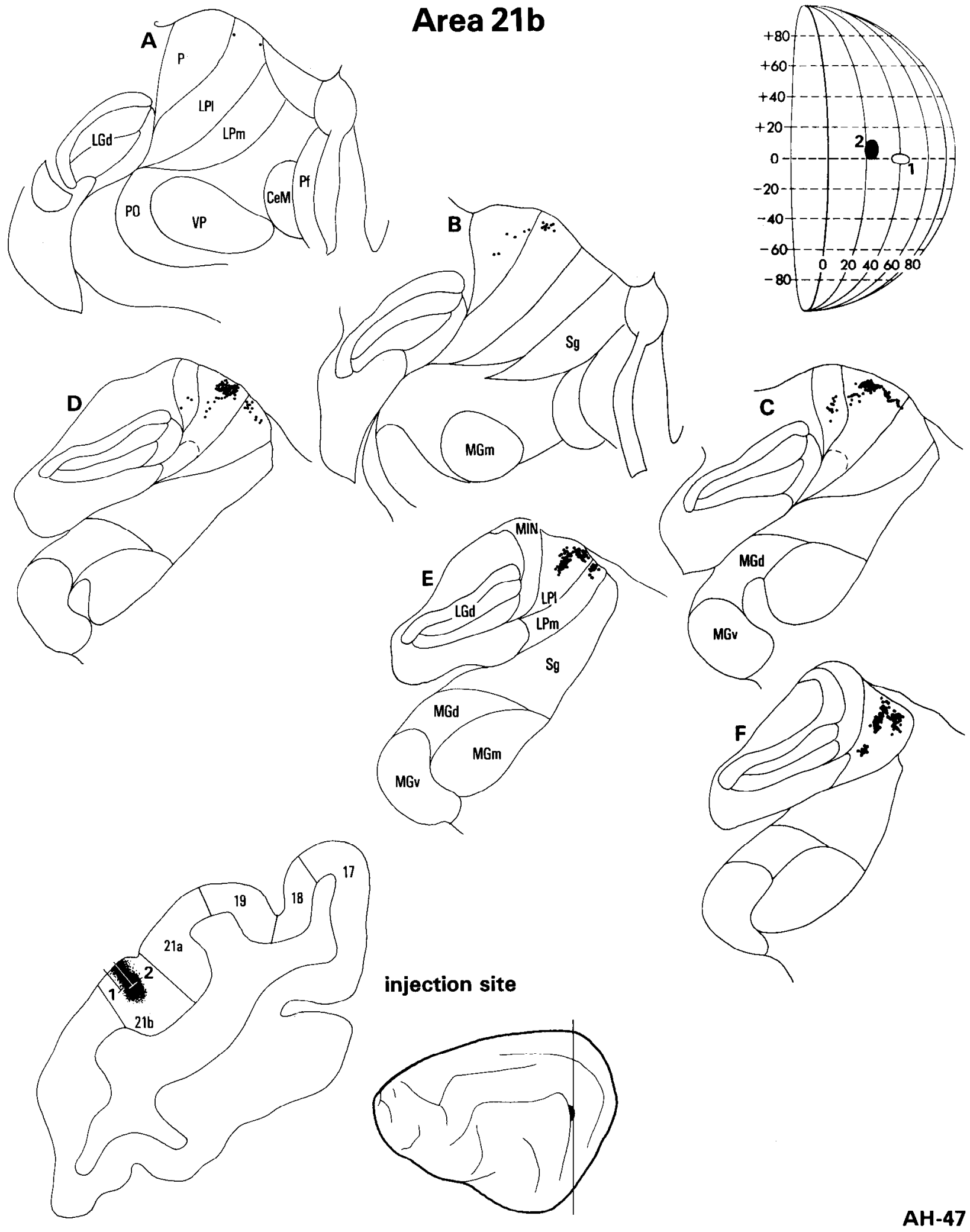

Figure 12. Chart of HRP-labeled cells in the cat's visual thalamus ( $A$ to $F$ ) after an HRP injection into cortical area 21 b. Two electrode tracks and recording sites ( 1 and 2 ), as well as the injection site, are illustrated in the coronal section of cortex. The receptive field recorded at points 1 and 2 are depicted on the perimetry chart. The injection was made at point 2 .

adjacent LPl is illustrated in Figures 20 and 21. In each structure, two populations of neurons, based on cell size, are clearly visible. A heavy focus of label appeared in the ventral and rostral portions of the I.Pl (Fig. 19). The
LPm and the major portion of the pulvinar nucleus were devoid of labeled cells. As in the previous case, the few HRP-labeled cells that were seen in the far rostral and lateral sector of the pulvinar nucleus are probably best 


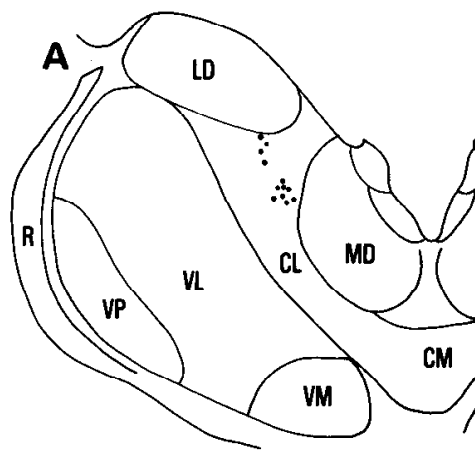

Area 19

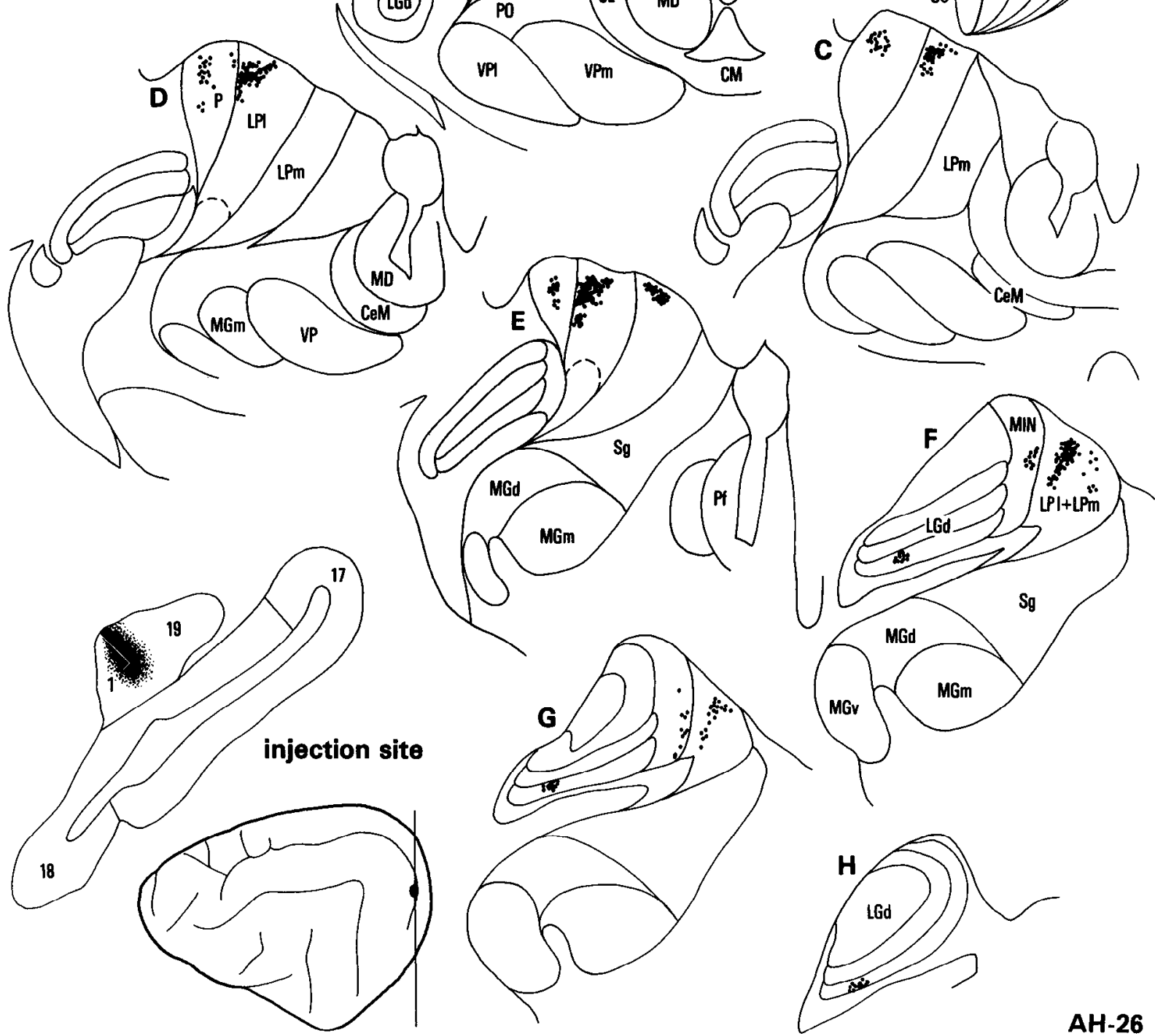

Figure 13. Chart of HRP-labeled cells in the visual thalamus $(A$ to $H)$ after an HRP injection into area 19 . The recording and injection sites are shown on the coronal section of the cortex. The receptive field of the injection site is depicted on the perimetry chart.

ascribed to the "geniculate wing." HRP-labeled cells were also evident in the CL and CM.

In summary, the three previous cases show that the representation of the lower visual field lies rostrally and ventrally in both subdivisions of the LP.

\section{Horizontal meridian}

The representation of the HM in the LP-pulvinar complex is best illustrated in Figure 22 which depicts an injection into area $20 \mathrm{a}$. In this case, the injection was centered at a representation $45^{\circ}$ away from the VM and $8^{\circ}$ below the HM, but label spread to include a region 20 to $50^{\circ}$ from the VM and from the HM to $10^{\circ}$ below. Thus, a $30^{\circ}$ long by $10^{\circ}$ wide representation of the $\mathrm{HM}$ was labeled. The retinotopic locus of injection was confirmed by the pattern of HRP (illustrated) and autoradiographic label in the parvocellular $C$ laminae of the LGd. Evidence that nearby area 19 was not included 


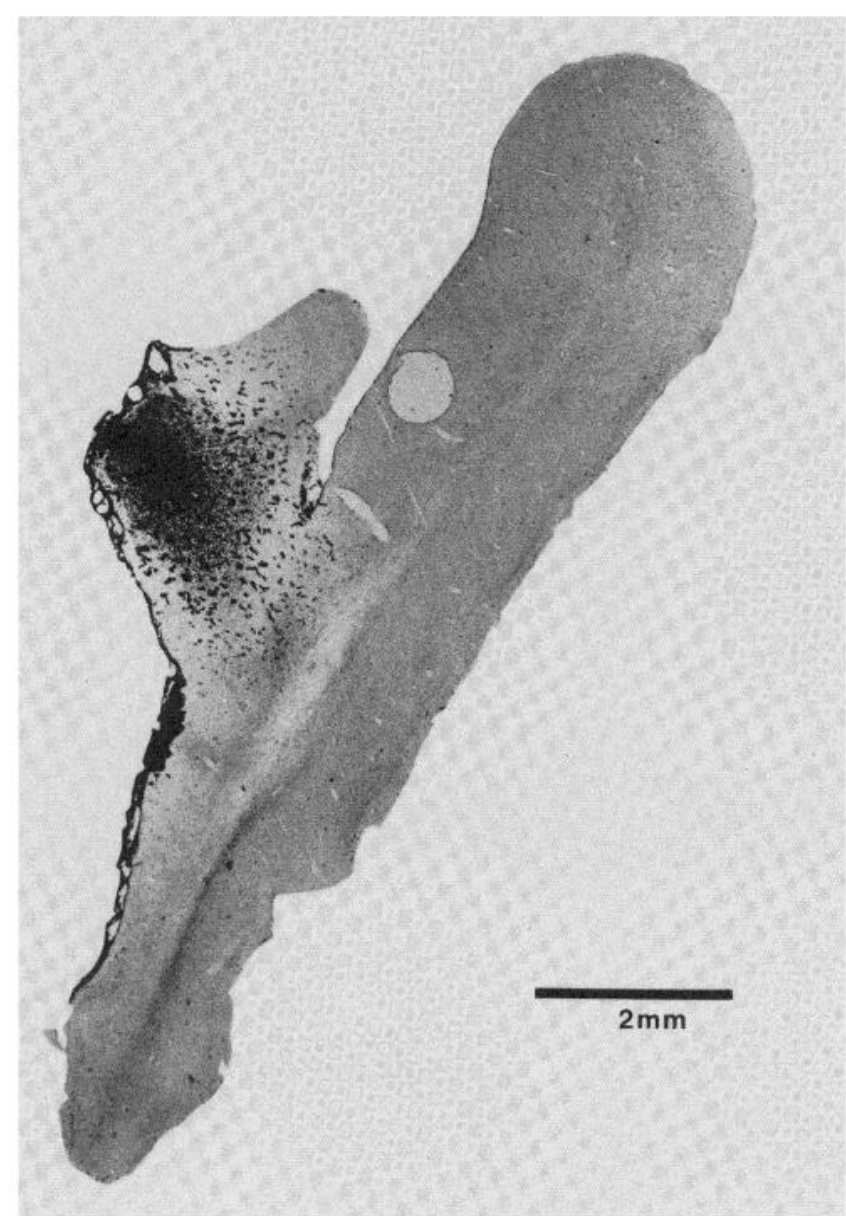

Figure 14. Brightfield photomicrograph of the HRP injection site shown in Figure 13.

within the injection site is shown by the absence of label in the magnocellular C layer and MIN of the geniculate complex. The label in the LP-pulvinar complex was located throughout much of its rostrocaudal dimension and in all three of its subdivisions. Caudally, the label was organized in two main bands, extending nearly the entire dorsoventral dimension of the LP-pulvinar complex. One strip was located in the LPl along its border with the pulvinar nucleus, and the other was located in the LPm along its border with the suprageniculate nucleus. At intermediate levels, the label in LPm diminished in size and was limited to the dorsal aspect of the nucleus. Additionally, label spread across the LPl-pulvinar border into the pulvinar nucleus. Rostrally, a predominate strip of label was present in the LPl. The great extent of the label suggests that a large area of the LPpulvinar complex is devoted to the representation of the HM. The CL and VA also contained HRP-labeled neurons.

\section{Visual periphery}

The same experiment (Fig. 22) also identified the location of the visual periphery representation in the LPpulvinar complex. On the basis of thalamic label, two representations are evident. One is located along the common border between the LPl and the pulvinar. The second is located at the medial edge of the LPm adjacent to the suprageniculate nucleus. Observe that the label in both the pulvinar and LPl extends further rostrally than does the label in the LPm.

Further confirmation for the finding that the representation of the visual periphery is located along both the LPl-pulvinar and LPm-suprageniculate borders was gathered from chartings of thalamic label following an injection into the visual periphery of area PMLS (Fig. 23). The location of HRP (illustrated) and autoradiographic label in the parvocellular C layers of the LGd and the adjacent MIN confirmed the retinotopic locus of the injection. In the extrageniculate visual thalamus, HRP label was seen predominantly in both subdivisions of the LP. Caudally, the label was distributed in two bands, one along the lateral edge of the LPl (see Fig. 24), the other along the medial edge of the LPm. Note that the patch of label ventral to the main band in the LPl (section $E$ ) appears to be related to the main band of $\mathrm{LPl}$ in section $D$. At intermediate levels, the band in the $\mathrm{LPm}$ disappears leaving only the strip in the LPl. Observe how the label in the LPl showed no change corresponding to the limits (dashed lines) of Rioch's posterior nucleus. The few labeled cells seen in the far rostral and lateral pulvinar nucleus are inconsistent with its retinotopy and are probably within the "geniculate wing" of the LGd complex.

In summary, the visual periphery is located along the common border of the LPl and pulvinar, and also along the medial border of the LPm. It extends throughout much of the rostrocaudal dimension of the complex.

\section{Ipsilateral fields}

The next experiment shows the effect of an injection at a retinotopic locus extending from the VM some $20^{\circ}$ into the ipsilateral field in area PLLS (Fig. 25). The label is focused in the LPm and extends medially from the common LPl-LPm border (VM representation) to include that portion of the LPm devoted to the representation of the visual field $15^{\circ}$ away from the VM in the contralateral visual field. This is consistent with the recent observation that many neurons in the LPm have ipsilateral receptive fields (Chalupa et al., 1981). As in other experiments in which the LPm contains thalamic label, the retrogradely filled neurons in this case tend to congregate into irregular patches. In this case, like that illustrated in Figure 5, we see a small focus of label in the LPl. The question of whether the LPl connects with area PLLS is a perplexing one since in several other cases (not illustrated) of area PLLS injections we failed to find label in the LPl. Symonds et al. (1981) in their thalamocortical autoradiography study concluded that the LPl does not have a projection to area PLLS. As Figure 25 shows, a significant amount of label was also present in the dorsal lateral sector of the suprageniculate nucleus, where it abuts the LPm. A focus of label was also observed in the dorsal division of the medial geniculate body. This pattern of thalamic label was also seen in other injections at areas along the banks at the middle suprasylvian cortex (Figs. 5 and 6), confirming earlier observations by Updyke (1981) and Winer et al. (1977). Additional thalamic label was present in the far rostral and lateral sector of the pulvinar. This sector appears to correspond to that zone which receives direct retinal 


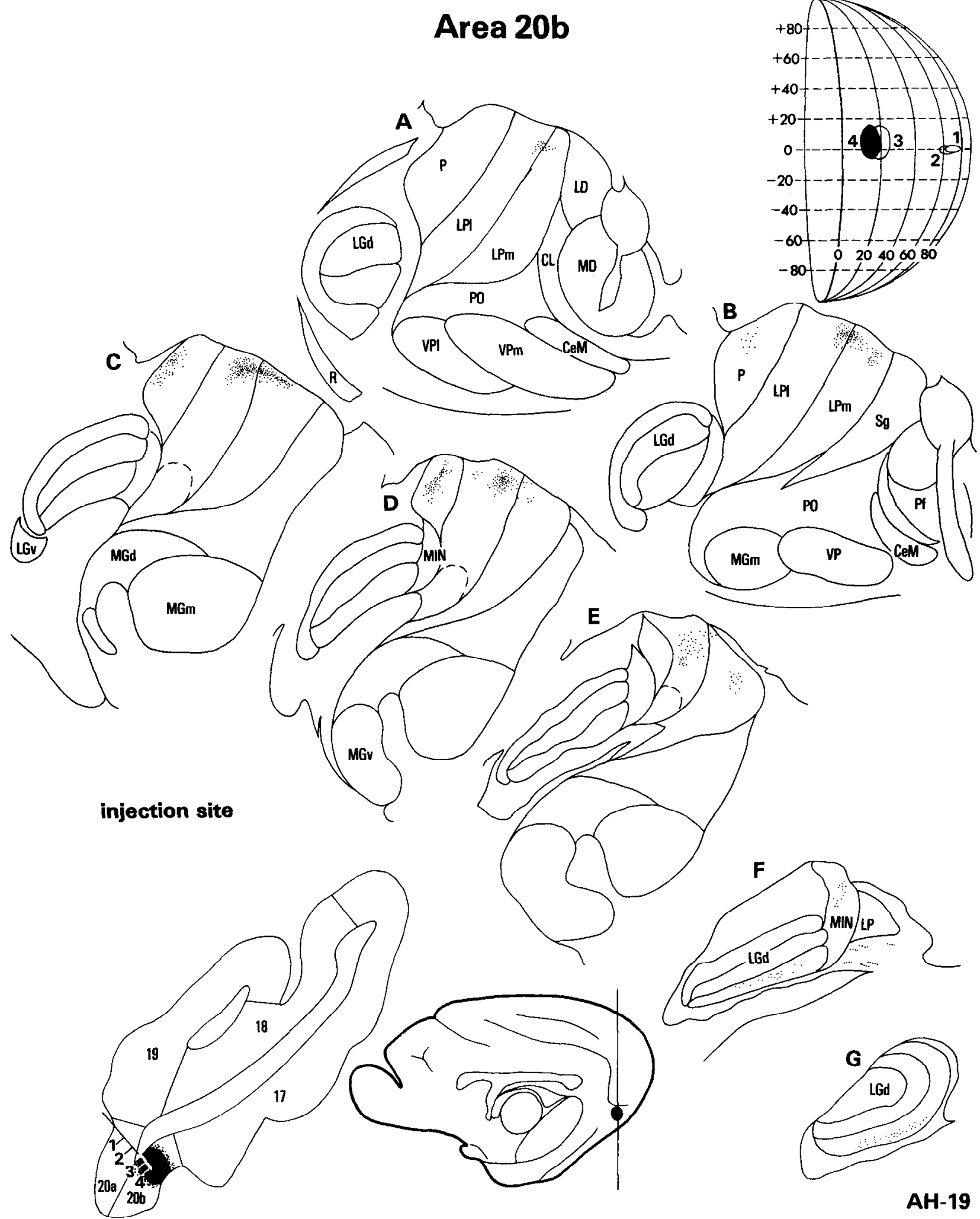

Figure 15. Chart of silver grains (black dots) in coronal sections of the thalamus $(A$ to $G)$ after an injection of tritiated leucine into area $20 \mathrm{~b}$. The electrode penetration and recording sites (1 to 4$)$ are depicted on the coronal section of the cortex. Receptive fields for each recording site are shown on the perimetry chart. The injection was made at point 4.

input (Fig. 3) and probably is best ascribed to the "geniculate wing." Finally, HRP-labeled neurons were observed in the intralaminar nuclei (CL and CM) and in the VA.

\section{Injections into other cortical areas}

An additional cortical region, which has been often associated with the visual cortex, is the parietal field, 


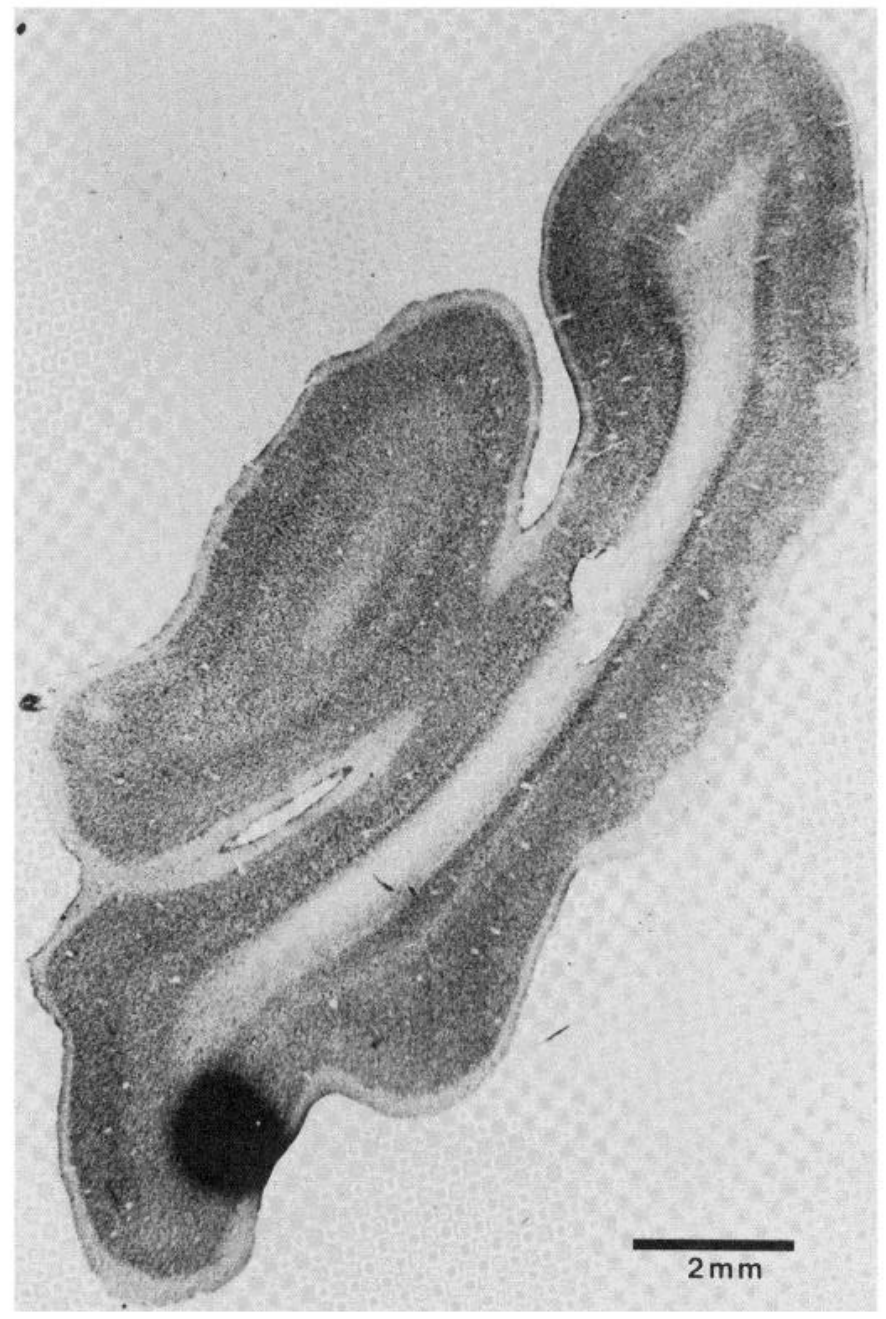

Figure 16. Brightfield photomicrograph of the injection site of tritiated leucine into area $20 \mathrm{~b}$ which is illustrated in Figure 15.

areas 5 and 7. Although many neurons in the area can be driven visually, no obvious retinotopy has been determined. We placed a relatively large HRP injection into the cortex, centered in the crown of the middle suprasylvian gyrus (Fig. 26). In the thalamus, a substantial projection was observed originating from the pulvinar nucleus. Most of the label (especially at caudal levels) was centered in the nucleus, avoiding its medial and lateral borders. At the rostral pole of the LGd, HRP-filled neurons were congregated in two clusters, one in the pulvinar nucleus, and the other coinciding with the caudal part of the lateral dorsal nucleus (LD). Finally, a substantial number of labeled cells were observed in the VA and CL.

Finally, one injection of tritiated leucine was made into the cingulate gyrus immediately below the splenial suclus (Fig. 27). Label was distributed widely throughout several thalamic subdivisions including the pulvinar nucleus and the LD, medial dorsal nucleus (MD), and VA. In addition, the cingulate gyrus was seen to connect with that medial thalamic region (Updyke's "LPm") that also connects with the sylvian gyrus (Fig. 2).

\section{Discussion}

In the present study we injected anterograde and retrograde tracers at electrophysiologically defined locations in 13 mappable visual areas and in adjacent fields in the cat cortex, and charted the patterns of label in the thalamus. These experiments support the view that the LP-pulvinar complex contains three main subdivisions, each containing a map of the visual field, and provide new details about the internal organization of these maps. Furthermore, when taken together, these experiments reveal the detailed relationships between each cortical visual area and each of the subdivisions of the LP-pulvinar complex. The knowledge of these relationships provides a basis for the functional grouping of cortical and thalamic areas and reveals a rich array of parallel routes by which visual information reaches the cortex.

\section{Retinotopy of the LP-pulvinar complex}

Because each cortical injection was localized by electrophysiological recording, and because injections were made in every visual area at different retinotopic loci, the patterns of projection yield an accurate picture of the extent and visuotopic organization in the LP-pulvinar complex. Foveal or VM representations lie along the lateral border of the pulvinar nucleus and along the LPl$\mathrm{LPm}$ border. By contrast, peripheral field representations are located on either side of the LPl-pulvinar border and along the medial edge of the LPm. These anatomical data also confirm the fact that upper fields are represented dorsally and caudally whereas lower visual fields are represented ventrally and rostrally. Thus, the extrageniculate visual thalamus contains three representations of the visual field and this observation compares favorably with previous findings (Mason, 1978; Raczkowski and Rosenquist, 1981; Updyke, 1977, 1981).

More specific conclusions regarding the representation of the visual field in the LP-pulvinar complex are also revealed by these experiments. Upper and lower field representations appear compressed when compared to the area of the LP-pulvinar complex devoted to fields near the HM. Thus, as in many visual cortical areas, the representation of the HM appears expanded compared to upper and lower visual fields.

We observed that cortical injections into the VM representation (e.g., Figs. 5, 8, 9, and 24) appeared to result in broader bands of label in LP-pulvinar than injections (e.g., Figs. 22 and 23) into peripheral field representations. This seeming exaggeration of VM representation based upon connectional data may be accounted for by the fact that most of the visual fields of cells in the LPpulvinar complex, even those centered quite peripherally, extend centrally to include the VM (Raczkowski and Rosenquist, 1981). Also, in two cases (Figs. 5 and 8) involving the VM we saw a discontinuous patch of label in the LPm ventral to the main projection. Whether this second focus indicates a new representation of the VM is unknown.

The LP-pulvinar complex has an unusual internal organization not previously described: a "point-to-plane" transformation of the visual field. This transformation 

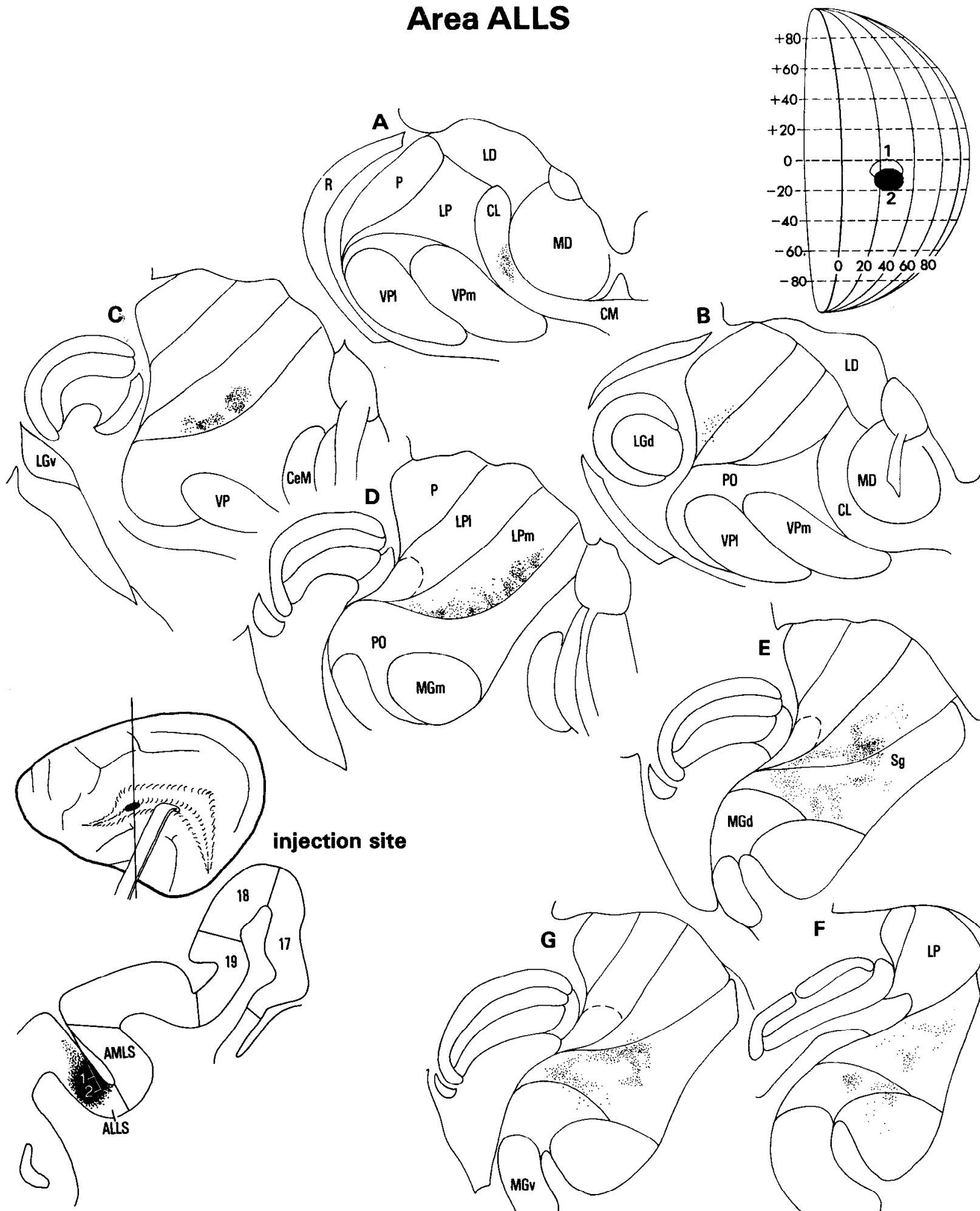

PO
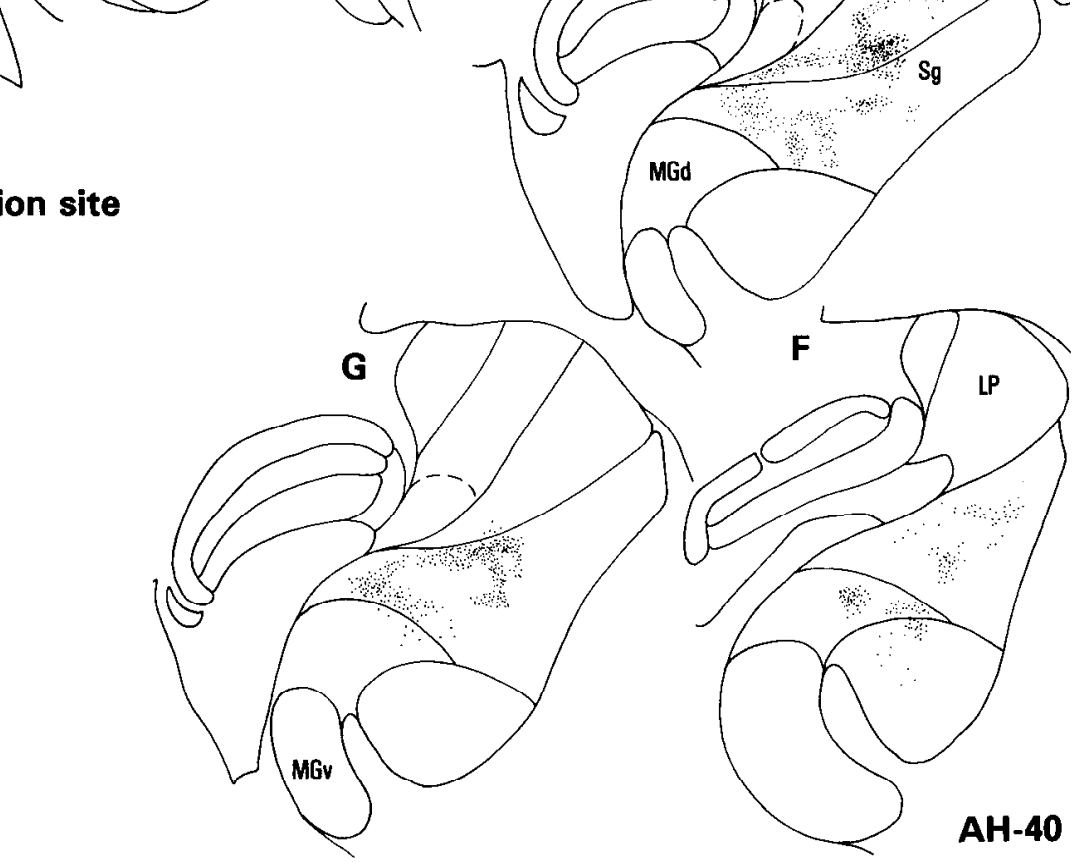

Figure 17. Charts of silver grains (black dots) in coronal sections of the visual thalamus $(A$ to $G)$ after an injection of tritiated leucine into cortical area ALLS. The coronal section of the cortex shows the electrode penetration and two recording sites ( 1 and 2). Receptive fields recorded at each recording site are depicted on the perimetry chart. The injection was made at point 2.

is suggested by the slab-like projection patterns of afferents and efferents. These slabs lie parallel to the borders between subdivisions. A peripheral field injection (e.g., Fig. 23) results in a robust slab of label extending dorsoventrally in the LPI through several coronal levels.
Thus, when viewed in three dimensions, a given subdivision of the LP-pulvinar complex can be visualized as a stacked set of planes, each representing a small portion of the visual field. This reflects a solution to the problem of how a two-dimensional space is represented in a three- 


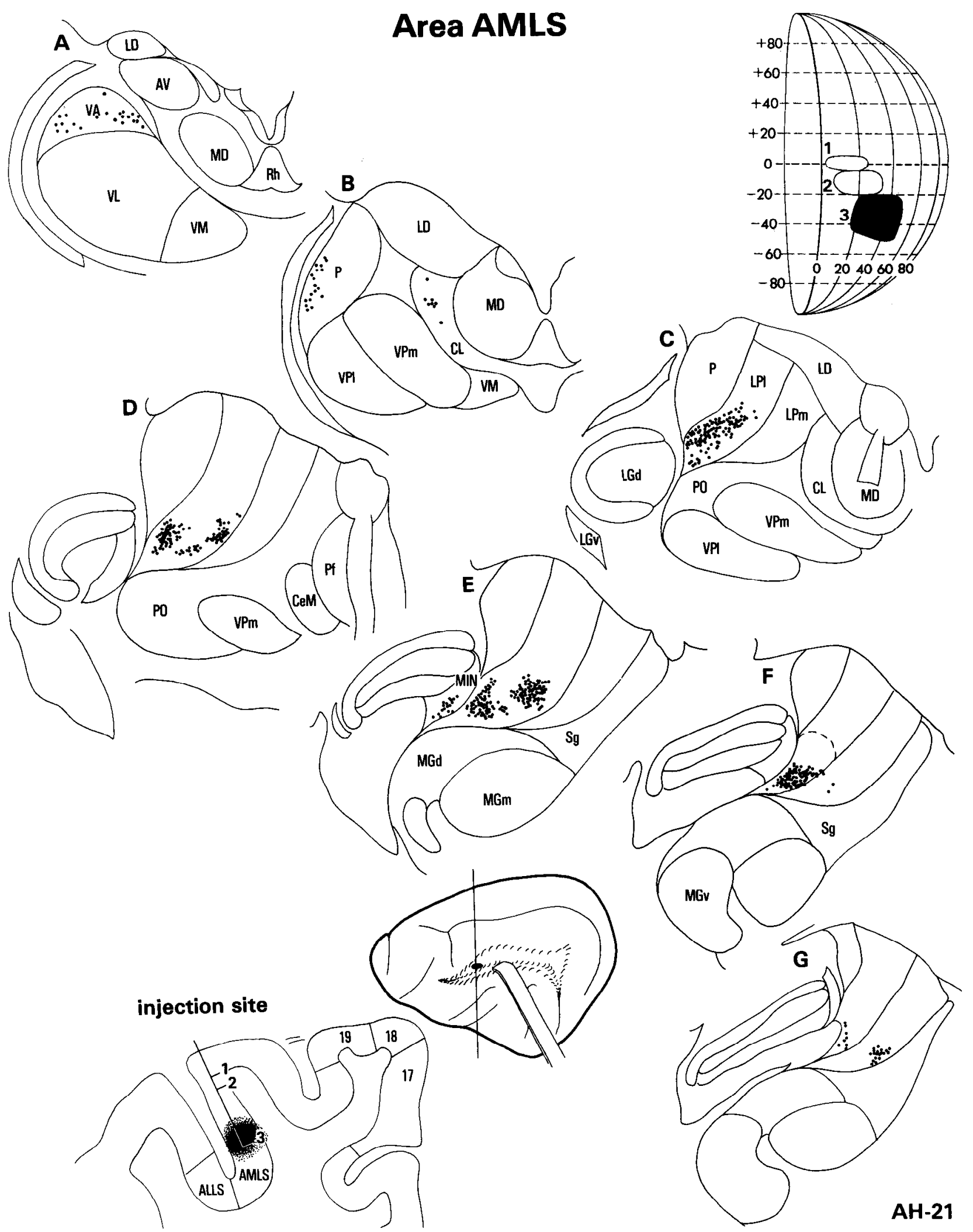

Figure 18. Chart showing HRP-labeled cells $(A$ to $G$ ) in the visual thalamus after injection of HRP into cortical area AMLS. The electrode track and three recording sites $\left(\begin{array}{ll}1 & \text { to }\end{array}\right)$ are shown on the coronal section of the cortex. Receptive field loci corresponding to the recording sites are illustrated in the perimetry chart. The injection was made at point 3.

dimensional nucleus. This problem and its solution appear to be unique to the LP-pulvinar complex. In the adjacent IGGd, for example, a first-order point-to-point transformation suffices. This reflects the fact that the LGd, when uncurled, looks remarkably like a two-dimensional slab of cortex (see Fig. 9; Sanderson, 1971). 


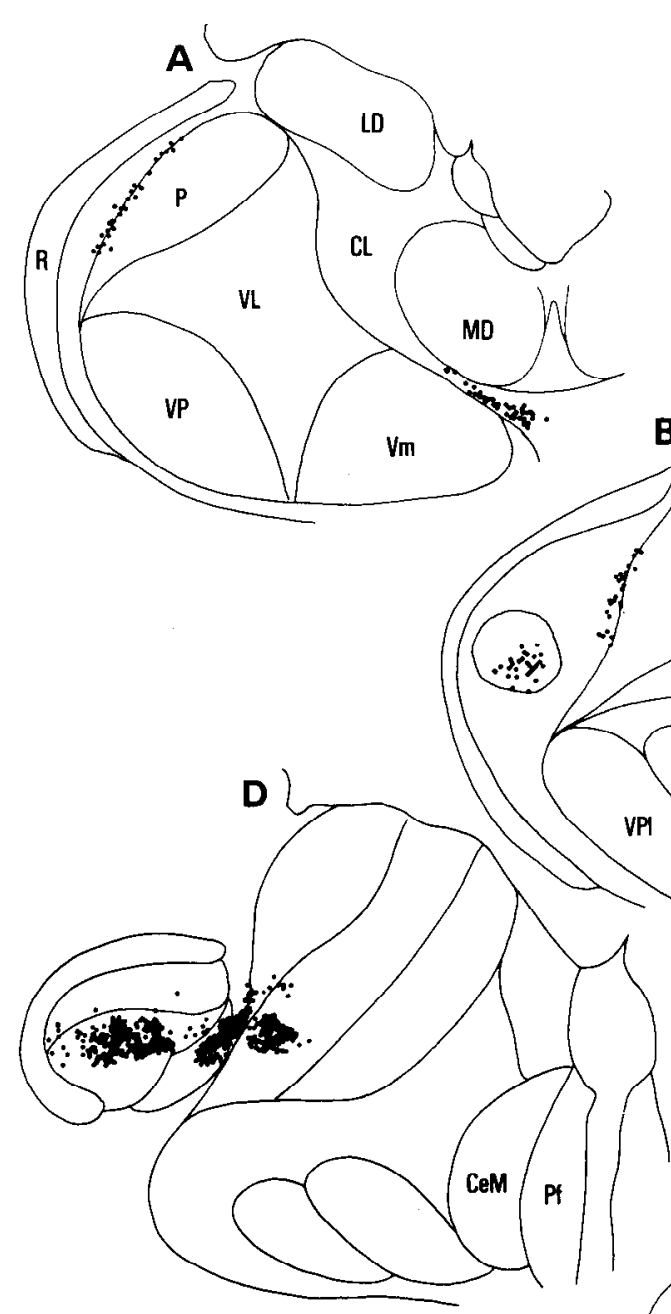

\section{Area 18}

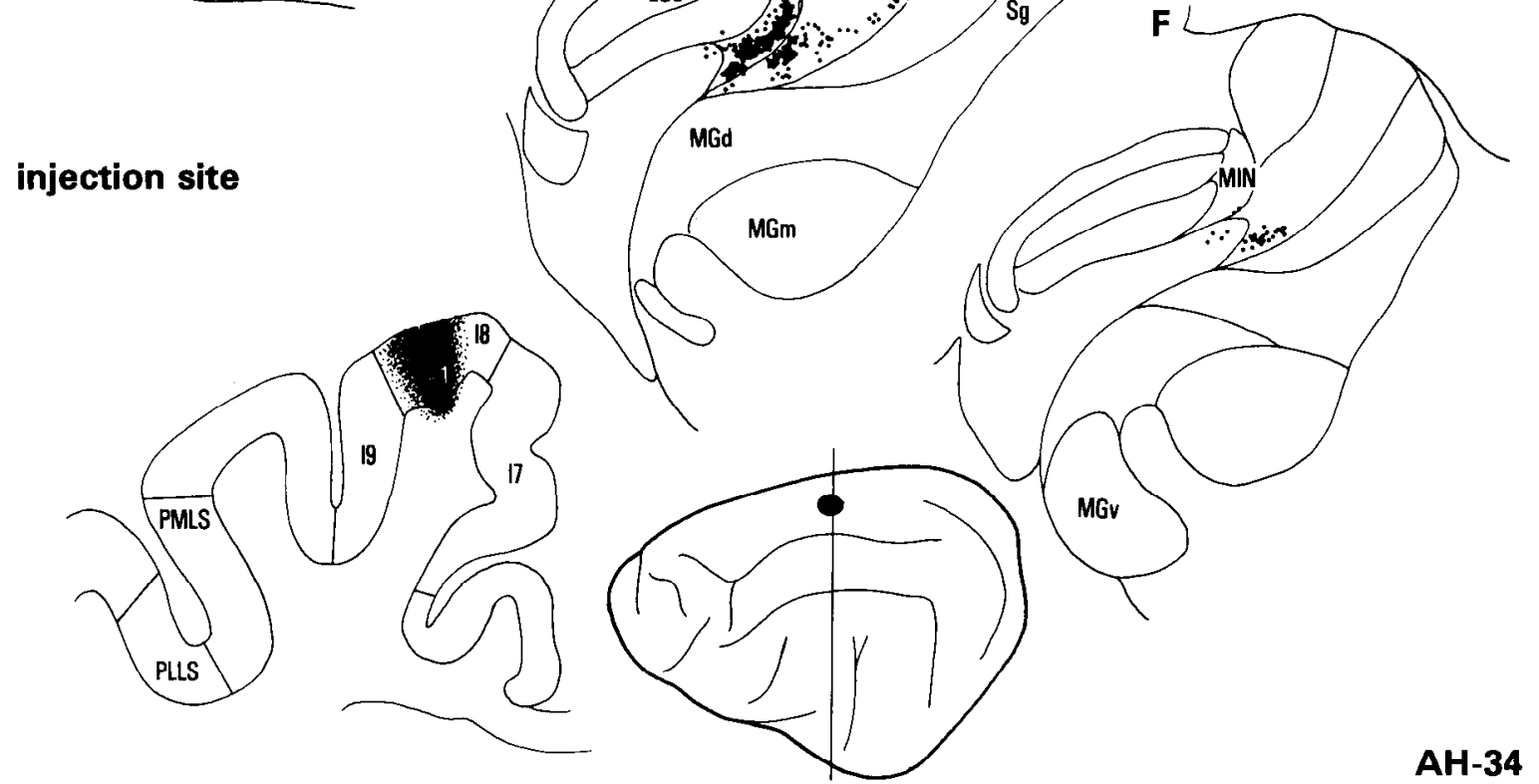

Figure 19. Chart of HRP-labeled cells in coronal sections ( $A$ to $F$ ) of the visual thalamus after an area 18 injection. The electrode penetration and the recording and injection sites are seen on the coronal section of the cortex. The receptive field recorded at the injection site is shown on the perimetry chart.

Relation between visual cortical areas and thalamic subdivisions

A major aim of this study is to describe the organization of extrageniculate thalamocortical circuitry in the cat. We relied on the principle of retinotopy not only for the parcellation of the thalamus, but also of the cortex. The reliance on this principle in subdividing the thalamus is amply confirmed by these connectional data. Since each of the three retinotopic zones of the LP- 


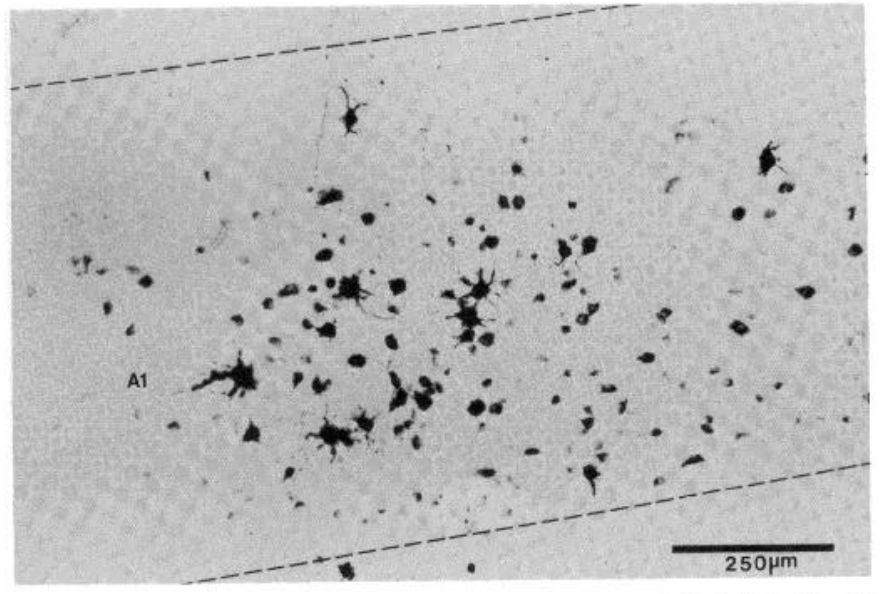

Figure 20. Brightfield photomicrograph of HRP-labeled cells in lamina $\mathrm{A} 1$ of the dorsal lateral geniculate nucleus after the area 18 injection illustrated in Figure 19.

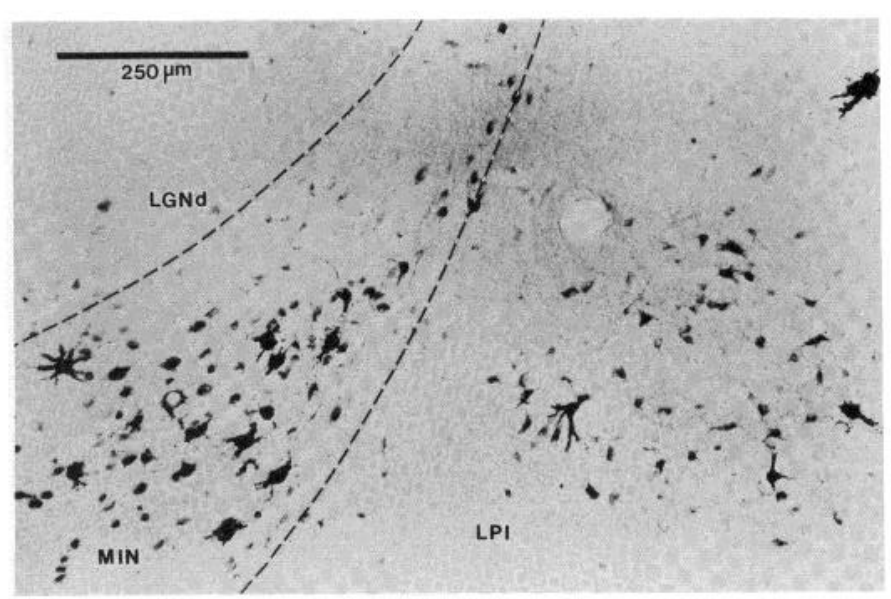

Figure 21. Brightfield photomicrograph of HRP-labeled cells in the MIN and LPl after the area 18 injection illustrated in Figure 19.

pulvinar complex have separate cortical targets (see also Rosenquist et al., 1974, 1982; Symonds et al., 1981), and since all of the visual cortex projects back to the thalamic region defined by the LP-pulvinar complex (see also Kawamura et al., 1974; Updyke, 1977, 1981) these three zones must compose the functional unit that is the extrageniculate visual thalamus.

Similarly, the retinotopic parcellation scheme of the cortex has been supported by these experiments. In every instance, the present mapping results confirm the features of retinotopy reported earlier (Palmer et al., 1978; Tusa et al., 1978, 1979, 1981; Tusa and Palmer, 1980). For example, in the lateral suprasylvian cortex, we confirmed the general retinotopic organization and observed that neurons in areas ALLS and AMLS were less reliably driven by visual stimuli than neurons in areas PLLS and PMLS. Anatomically, the posterior areas in the lateral suprasylvian cortex (PMLS, PLLS, VLS, and DLS) are interconnected with the parvocellular $\mathrm{C}$ layers of the LGd whereas the anterior areas are not (see also Raczkowski and Rosenquist, 1980; Marcotte and Updyke, 1981; Tong et al., 1982).

Few studies attempt to relate both the cortical parcel- lation into functional areas and retinotopic considerations within an area to the problem of extrageniculate thalamocortical connectivity. Where comparisons are possible, however, our findings are consistent with and confirm conclusions reached by others. Earlier anterograde degeneration studies (Graybiel, 1972b; Niimi et al., 1974) suggested a division of the LP-pulvinar complex into three zones, on the basis of their largely nonoverlapping projections to the cortex. These studies concluded that the pulvinar nucleus projects onto the crown of the middle and posterior suprasylvian gyri and the splenial sulcal area. The lateral division of the LP projects to the cortex along the medial bank of the middle suprasylvian sulcus and to the posterior suprasylvian gyrus. The medial division of the LP innervates the lateral bank of the middle suprasylvian sulcus and its continuation in the posterior suprasylvian sulcus.

In a recent study from this laboratory (Symonds et al., 1981) tritiated amino acid injections were made into each of the three thalamic subdivisions in order to reveal their cortical targets. Attempts were made to relate the pattern of cortical label to cortical areas on the basis of "standard" retinotopic maps. Although the conclusions of that study correspond closely to those reported here, the use of "standard" maps is potentially risky since identification of boundaries between areas is not trivial and may vary from animal to animal. The pulvinar nucleus sends axons to retinotopically organized areas $19,20 \mathrm{a}$, and $20 \mathrm{~b}$, as well as to areas with poorly understood retinotopy such as the cingulate gyrus, the splenial visual area, and areas 5 and 7 . The lateral division of the lateral posterior nucleus projects to areas $17,18,19,20 \mathrm{a}$, PMLS, and VLS. Finally, the medial division of the LP projects onto areas $20 \mathrm{a}$, DLS, PLLS, and the posterior suprasylvian area (PS) of Heath and Jones (1971). Despite the problems of identifying cortical areas of termination using "standard" maps, all of the connections reported by Symonds et al. (1981) have been confirmed by the present study. However, by employing receptive field mapping methods along with HRP and tritiated leucine injections, we were able to identify additional connections not demonstrable using the methods of Symonds et al. (1981) and others.

Figure 28 shows a summary of thalamocortical interrelationships that are based upon combining the results of the present study with those previously carried out in this laboratory (Rosenquist et al., 1974; Symonds et al., 1981). It is important to emphasize that this figure depicts only connections that are robust and reproducible in every case. The combined results shown in Figure 28 suggest that cortical areas may be grouped into functional sets on the basis of sharing a common pattern of thalamic input. The top-most box contains the cortical areas that receive thalamic input solely from the pulvinar nucleus. These results are derived from Symonds et al. (1981) but are confirmed for the cingulate cortex and areas 5 and 7 by our cases illustrated in Figures 26 and 27.

The next box in Figure 28 shows that areas 17 and 18 both receive input solely from the lateral division of the LP. This finding is illustrated in the cases seen in Figures 4 and 19 and has been confirmed by many other laboratories (e.g., Graybiel, 1972b; Rosenquist et al., 1974; 


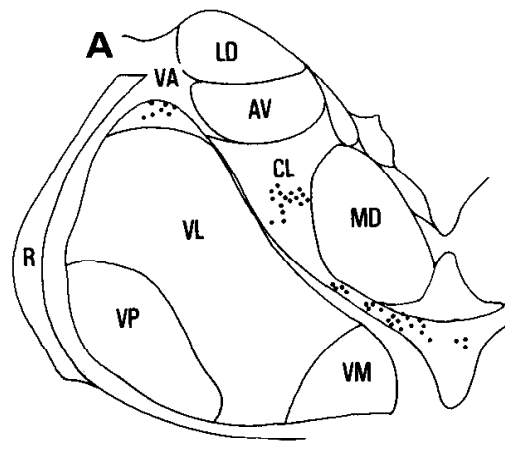

\section{Area 20a}

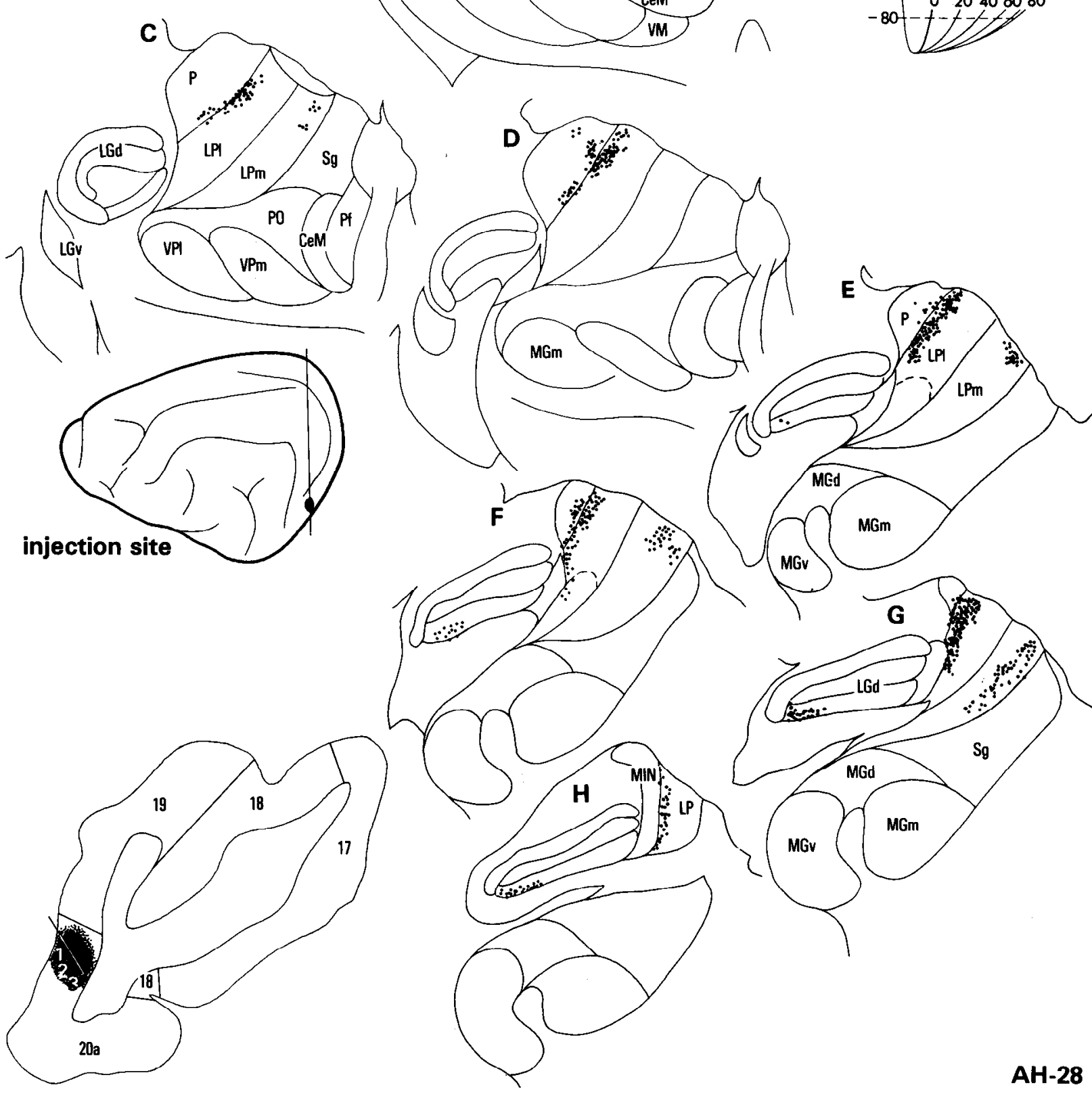

Figure 22. Chart of HRP-labeled cells in coronal sections ( $A$ to $H$ ) of the thalamus after an HRP injection into area 20a. The electrode track, recording site, and injection site are seen on the coronal section of the cortex. The receptive fields of cells encountered along the electrode penetration are shown in the perimetry chart. The injection was made at point 1.

Maciewicz, 1975; Hollander and Vanegas, 1977; Updyke, 1977; Hughes, 1980; Symonds et al., 1981).

The third box in Figure 28 contains five cortical visual areas, 19, 20a, 20b, 21a, and 21b (see Figs. 8, 12, 13, 15, and 22), each of which receives thalamic input from all three subdivisions of the LP-pulvinar complex. With the exception of the connections with area 19, there is relatively little in the literature with which to compare these 


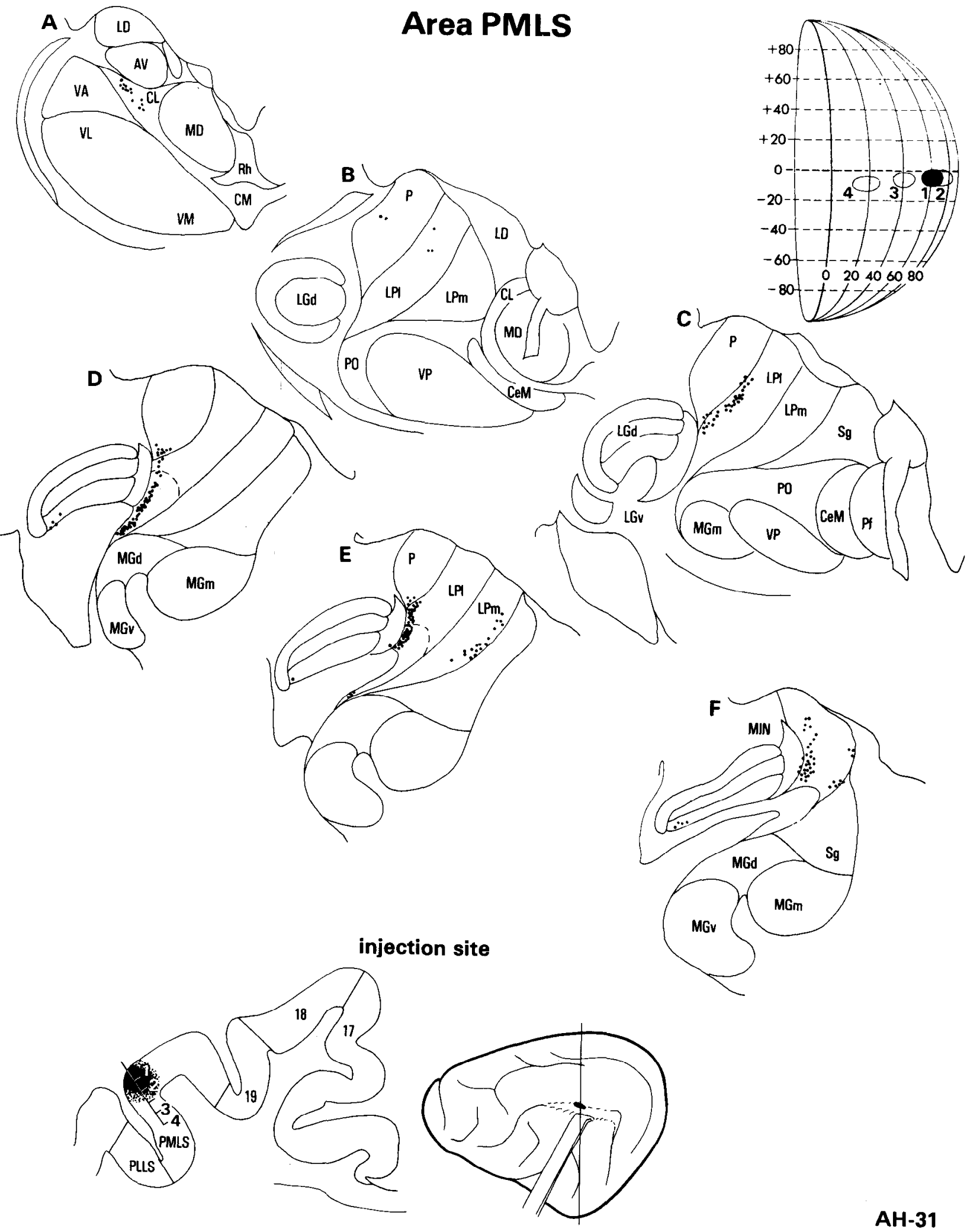

Figure 23. Chart of HRP-labeled cells in coronal sections of the thalamus ( $A$ to $F$ ) after an HRP injection into area PMLS. The coronal section of cortex shows the electrode track, recording sites, and the injection site. The receptive fields recorded at each of the numbered points along the electrode track are depicted on the perimetry chart.

results. Previous studies tracing projections from thalamus to cortex have documented a connection from both the LPl and the pulvinar nucleus to area 19 (e.g., Graybiel, 1972b; Symonds et al., 1981). The connection with the LPm, however (see Fig. 13), has been observed in only one other study (Updyke, 1977). However, the fact that we identified area 19 electrophysiologically prior to injection, used a very sensitive HRP chromogen, and 


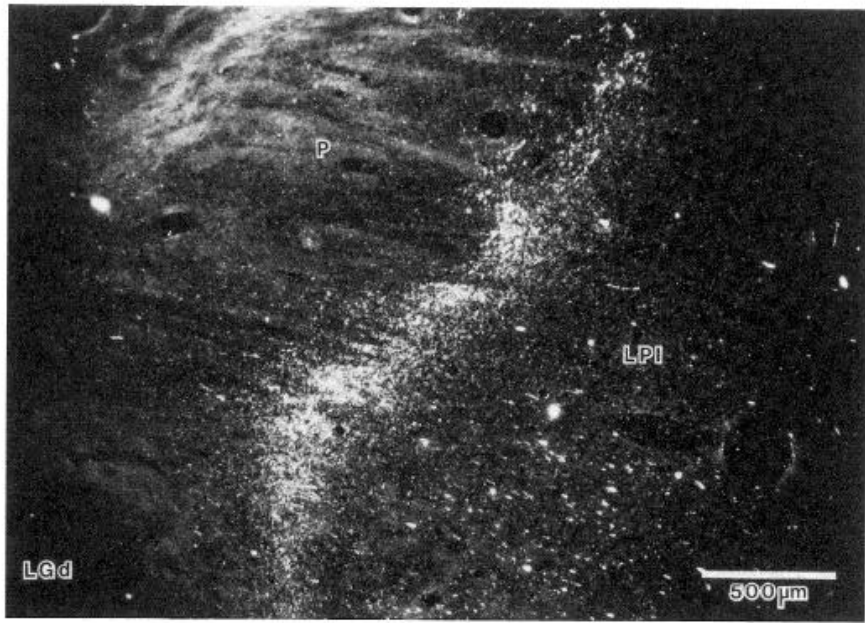

Figure 24. Darkfield photomicrograph of silver grains in the cat LP-pulvinar complex after an injection of tritiated leucine into area PMLS. This is the same case as illustrated in Figure 23 where HRP label is charted.

verified these connections in other cases, lends confidence to the presence of a connection between the LPm and area 19. Symonds et al. (1981) observed that area 20 a received projections from all three thalamic subdivisions but did not report projections to areas $21 \mathrm{a}$ and $21 \mathrm{~b}$ following injections into the tectorecipient sector of the LP. Again, these differences can be attributable to methods of identifying cortical areas as well as to the tracer employed.

The fourth box in Figure 28 illustrates three cortical visual areas, PMLS, AMLS, and VLS, (see Figs. 9, 11, 18 , and 23) that receive input from both the LPl and the LPm. The connection between areas AMLS and PMLS with the LPm is rather sparse and, not surprisingly, it was not uncovered by injection of amino acids into the LPm (Symonds et al., 1981). However, Updyke (1981) does illustrate a sparse projection from the medial bank of the suprasylvian sulcus to the tectorecipient thalamic sector.

The fifth and bottom box in Figure 28 shows four cortical areas, ALLS, PLLS, DLS, and PS (see Figs. 5, 17 , and 25), whose exclusive or primary input from the LP-pulvinar complex arises from the LPm. These findings are consistent with the results previously reported by Symonds et al. (1981) and Updyke (1981).

As Figure 28 emphasizes, cortical visual areas can be grouped, by virtue of their connections with particular subdivisions of the LP-pulvinar complex, into several clusters. Interestingly, each of the cortical areas within a box occupies a given longitudinal strip in the visual cortex which is contiguous with one or more of the other members of that group. Moreover, as one reads down the chart, there exists a lateral to medial progression in the LP-pulvinar complex and a reverse medial to lateral progression in the cortex. Thus, the most lateral subdivision of the extrageniculate visual thalamus (pulvinar) is connected with the most medial areas of the cortex: cingulate gyrus and splenial visual area. Progressively more medially located thalamic zones are connected with more laterally situated cortical areas. Similar relation- ships have been reported among connections of the lateral, anterior, and medial thalamic nuclei with the frontal cortex in the primate (Kievet and Kuypers, 1977).

\section{Parallel pathways}

In addition to the geniculostriate route, a number of parallel pathways carry visual information to the multiple visual cortical areas. For example, ascending inputs from the midbrain and the striate cortex terminate in essentially parallel, nonoverlapping zones within the LP. pulvinar complex. However, until now, it has not been clear how input-output relationships between the LP. pulvinar complex and each of the extrastriate visual areas fit into this scheme. As can be seen in Figure 28, there exist in the extrageniculate pathway several reasonably distinct channels by which visual information from the retina is routed to the visual cortex. The target of the superficial layers of the superior colliculus, LPm, is robustly connected with areas ALLS, PLLS, DLS, and PS, whereas the target of the striate cortex, LPl, sends projections especially to areas AMLS, PMLS, and VLS. The target of the pretectum (pulvinar) is especially connected with areas $19,5,7$, and the splenial visual area and the cingulate gyrus.

Because each of the thalamocortical pathways summarized in Figure 28 is reciprocal, there is a potential for great interaction among these "distinct" parallel channels and sets of cortical areas. For example, cortical areas 17 and 18 receive input from the LGd complex and $L P l$, but, through corticothalamic pathways, projections from these areas back to the LPl can potentially influence a vast expanse of visual cortex; namely, those areas receiving from the LPl. Furthermore, corticothalamic connections from the targets of the LPl can potentially influence cells in the pulvinar and LPm and all of their targets: i.e., virtually all visual cortical areas thus far discovered.

Thus, when overlapping connections between parallel pathways within the extrageniculate thalamus are considered, thalamocortical and corticothalamocortical pathways take on added meaning. They serve to extend the sphere of influence of key incoming inputs. Essentially, tectal, striate, and pretectal influences can, via thalamocortical interconnections alone, reach all cortical areas. In this fashion, corticothalamic-cortical pathways take on the role traditionally reserved for intrinsic cortical connections - that of associating inputs from several cortical areas. Similarly, these connections suggest that the LP-pulvinar complex is more closely aligned with the visual cortex than with sensory relay nuclei such as the LGd (see also Graybiel and Berson, 1981). Perhaps the LP-pulvinar nucleus may best be described as an association nucleus, a role ascribed to it many years ago by Rose and Woolsey (1949).

The fact that individual zones in the extrageniculate visual thalamus project to multiple cortical areas suggests that individual cells within a given zone of the LP. pulvinar complex may project to more than one cortical area. We have confirmed this hypothesis using newly developed fluorescent retrograde tracer methods (Kuypers et al., 1980). We injected the fluorescent dyes fast blue and Nuclear Yellow into two separate cortical areas and observed their retrograde transport to the thalamus 

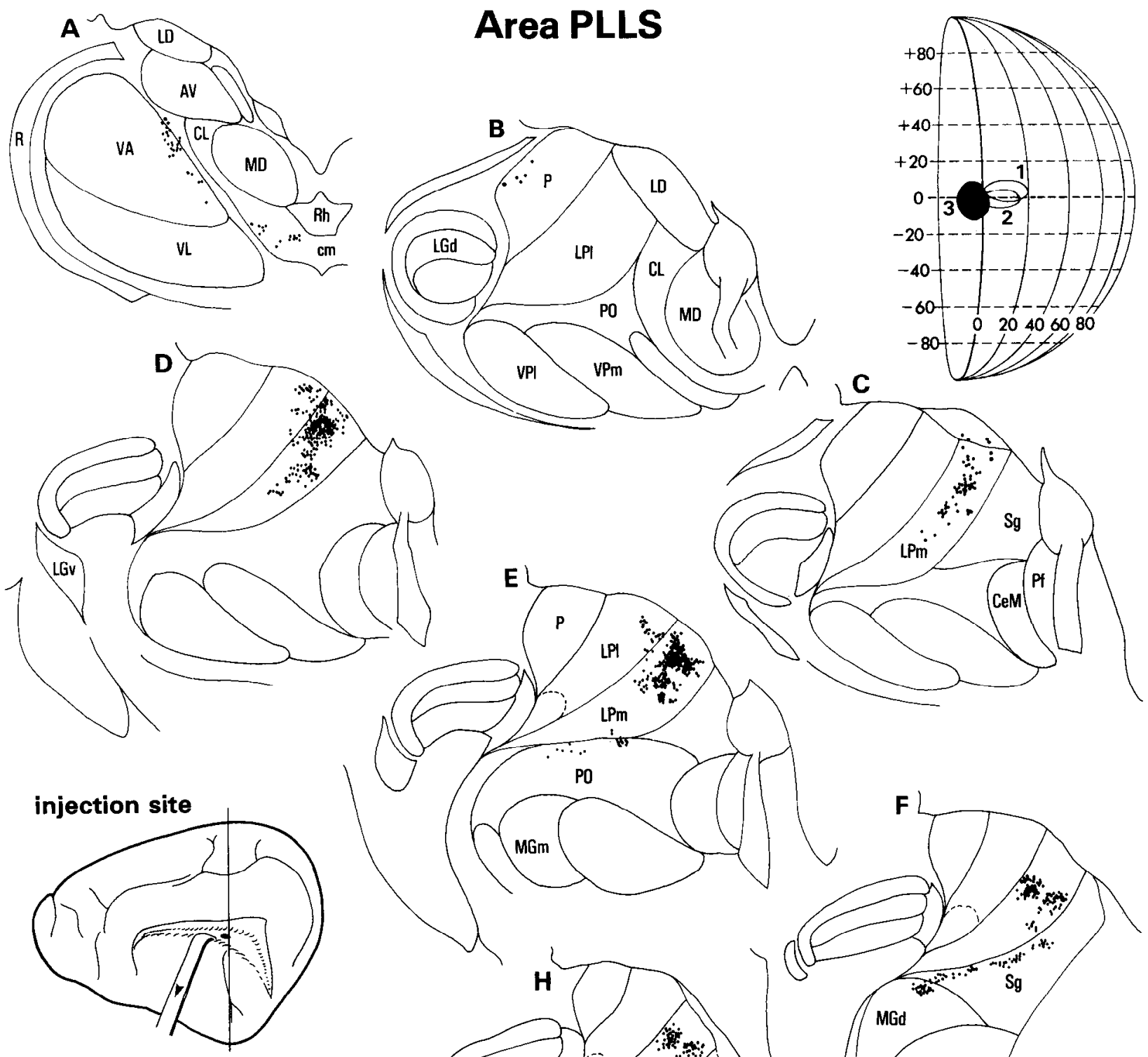

injection site

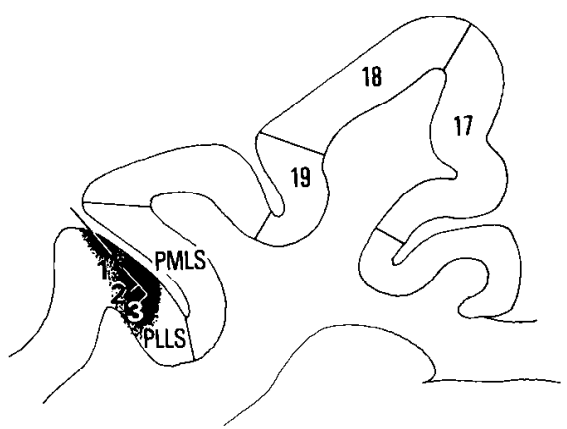

Area PLLS 


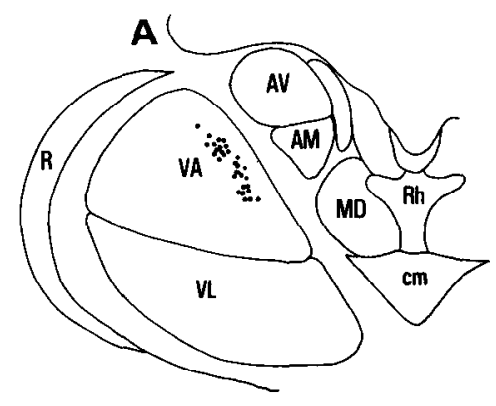

\section{Area 5-7}
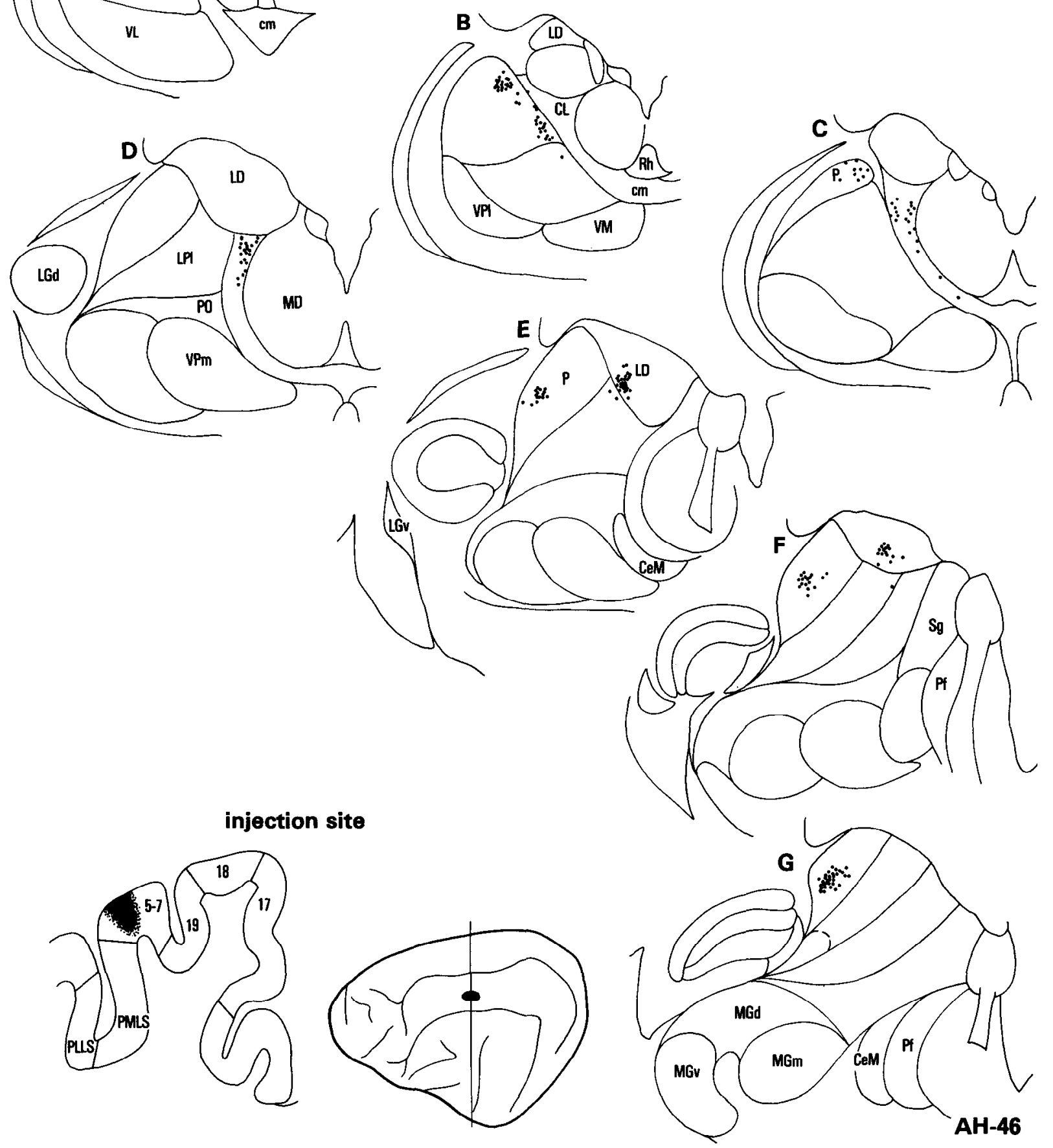

Figure 26. Chart of HRP-labeled cells in coronal sections of the thalamus ( $A$ to $G$ ) after an HRP injection into areas 5 and 7 of the cortex. The injection site is seen on the coronal section of the cortex and on the dorsolateral view of the brain. No recordings were made in this experiment.

(Kaufman et al., 1982). We have found double-labeled cells in both the pulvinar and LPl when two of their cortical targets were injected.

Significance of widespread, multiple visual pathways

To understand why such a complex array of multiple visual pathways exists in the extrageniculate visual sys- tem, we must determine what information is processed through these routes and what functions are performed by the cortex innervated by these pathways. Both the geniculocortical and extrageniculocortical visual systems are comprised of parallel channels, designated as $\mathrm{W}, \mathrm{X}$, and $Y$ cell pathways (see Stone, et al., 1979, for a review). In the geniculocortical system, where those pathways 


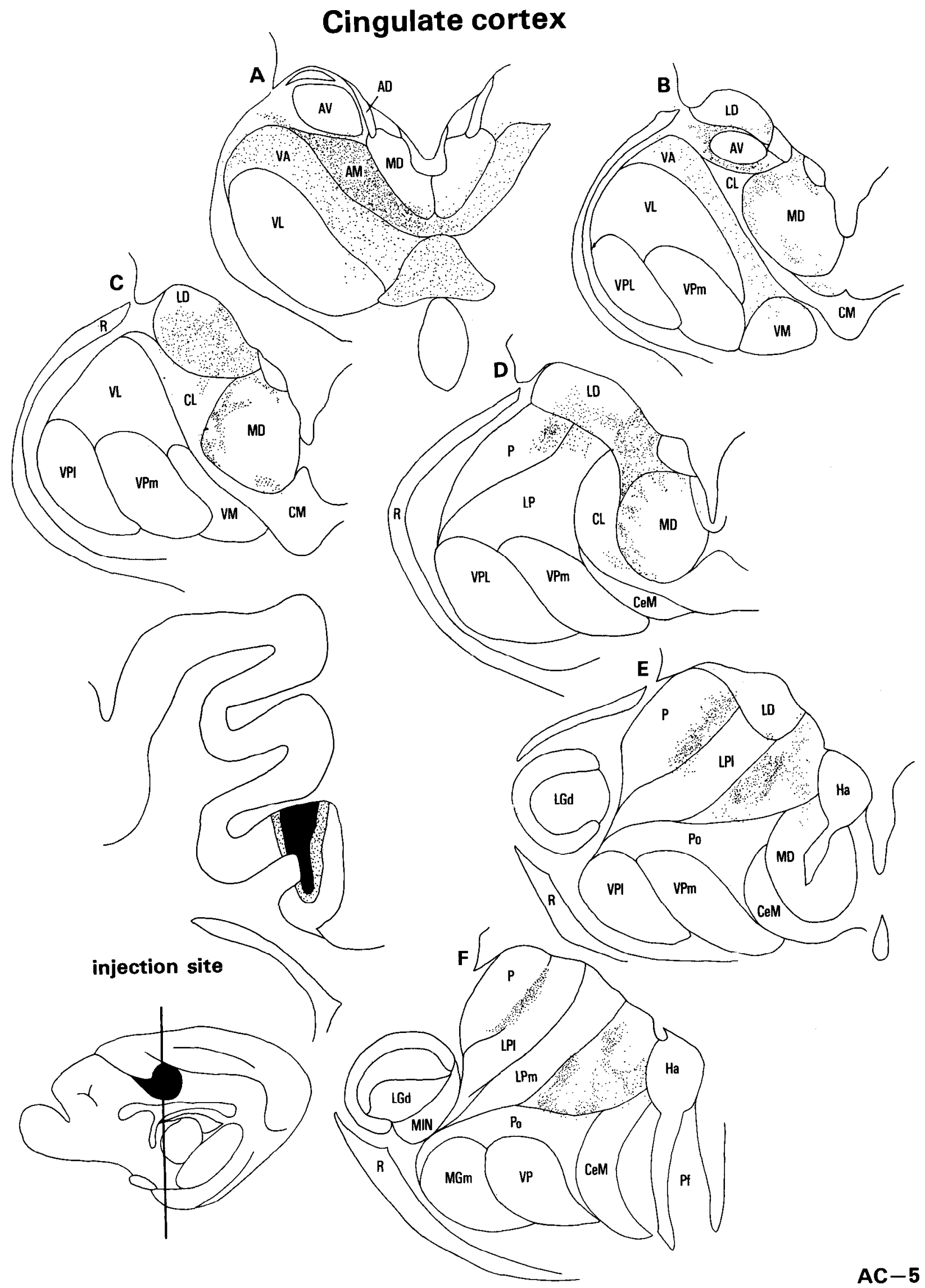

Figure 27. Charts of silver grains (black dots) in coronal sections ( $A$ to $F$ ) of the cat's thalamus after injection of tritiated leucine in the cingulate gyrus. 


\section{Thalamocortical Connections in the Cat}

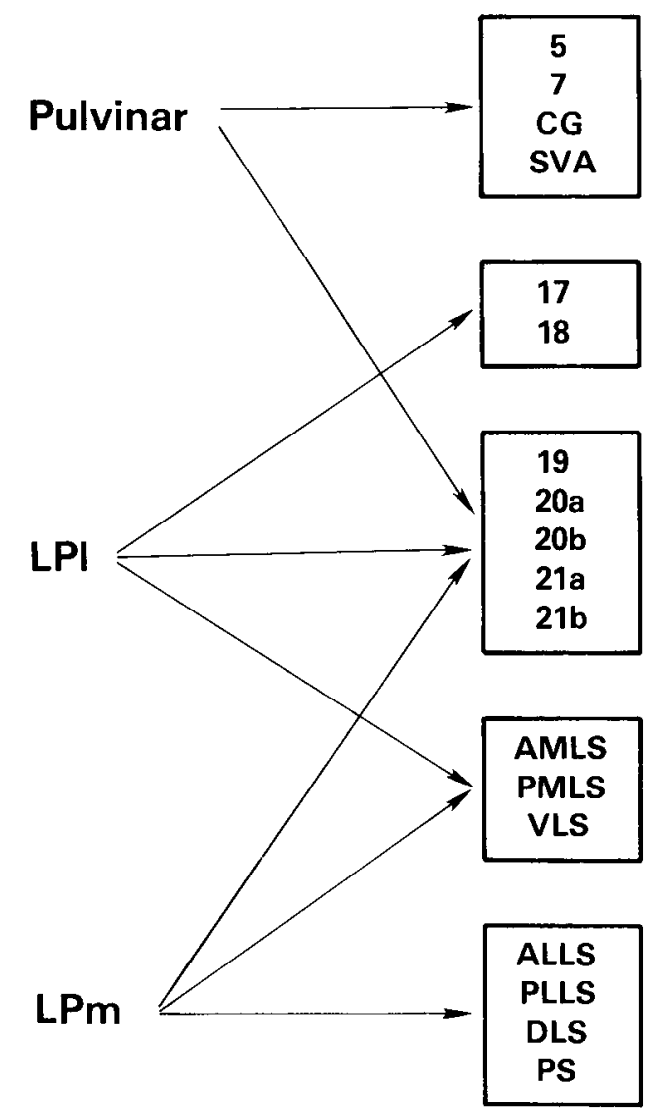

Figure 28. Summary chart of thalamocortical connections in the cat. Cortical areas within each box receive an identical pattern of input from the LP-pulvinar complex.

were first discovered, all three cell channels exist. In the A laminae, $\mathrm{X}$ and $\mathrm{Y}$ cell types are found and these cells project exclusively to areas 17 and 18 (Stone and Dreher, 1973; LeVay and Ferster, 1977). The C laminae and the adjacent MIN contain W and Y cells, and these nuclei have widespread projections to multiple cortical areas beyond areas 17 and 18 (Wilson et al., 1976; Dreher and Sefton, 1979; Marcotte and Updyke, 1981; Raczkowski and Rosenquist, 1981; Stanford et al., 1981; Tong et al., 1982).

In the extrageniculate cortical visual system, these pathways are less firmly established, but a growing body of evidence suggests that mainly $\mathrm{W}$ and $\mathrm{Y}$ cell pathways relay through this system. In the cat, subcortical afferents to the LP-pulvinar complex arise predominantly from cells in the nucleus of the optic tract (Berman, 1977) and the lower half of the superficial gray layers of the superior colliculus (Kawamura and Kobayashi, 1975; Graham, 1977; Kawamura et al., 1980). Some cells in the upper half of the superficial layers also project to the LP complex (Caldwell and Mize, 1981). From measurements of conduction velocity, $\mathrm{W}$ (direct) and $\mathrm{Y}$ (indirect) pathways have been identified in the nucleus of the optic tract (Hoffman and Schoppmann, 1975). In the colliculus, $\mathrm{W}$ cells have been encountered in the upper superficial gray layer, whereas $\mathrm{Y}$ cells are more likely to be encountered in the vicinity of the optic layer (Hoffman, 1973). When HRP is restricted to the upper superficial gray layer, the smallest ganglion cells are labeled, whereas injections confined to the lower superficial gray layer label mainly the largest ganglion cells (Itoh et al., 1981).

Significantly, no X cell pathways have been firmly established in the extrageniculate visual system. Whereas X cells comprise about one-half of all retinal ganglion cells, their cortical projection (via the LGd) is limited to area 17 (Stone et al., 1979). By contrast, W and $\mathrm{Y}$ cells together account for one-half of retinal ganglion cells, but, owing to LP-pulvinar-cortical pathways (as well as pathways through the $\mathrm{C}$ laminae and MIN of LGd), they may influence a large number of extrastriate cortical areas. The functional significance of this relative increase in the cortical domain of $\mathrm{W}$ and $\mathrm{Y}$ pathways may be suggested by ablation-behavior, psychophysical, and neurophysiological evidence. Cats with bilateral lesions of area 17 and most of area 18, which remove all of the $\mathrm{X}$ cell cortical projection system, have remarkably good vision (Berkley and Sprague, 1979). Only on tests of spatial acuity are these animals deficient. These data argue that discrimination of pattern and shape is mediated by one or more pathways in the tectothalamocortical system and/or the extrastriate part of the geniculocortical pathway, the systems through which apparently only $\mathrm{Y}$ and $\mathrm{W}$ cell pathways travel (see also Sherman, 1982).

Furthermore, when contrast sensitivity functions are obtained for $\mathrm{X}$ and $\mathrm{Y}$ cells, one major difference emerges (Lehmkuhle et al., 1980). Y cells are relatively sensitive to low spatial frequencies and $\mathrm{X}$ cells are not. Interestingly, considerable information about form vision is contained in these low frequencies (for a recent review, see Sherman, 1979).

Although all mammals have the capacity to perceive form, seemingly, some animals like the hedgehog perform this task in a cruder fashion than does a cat or monkey (Diamond and Hall, 1969). All higher mammals have multiple representations of the visual field, whereas primitive mammals have only two (for a recent review, see Kaas, 1978). Thus, in the course of evolution, the capacity to perceive objects has been refined and this refinement has been reflected in brain evolution by the increased number of visual cortical areas and the pathways innervating them. As more cortical areas arise, the connections among them are altered, providing a morphological basis for further specialization and refinements in the ability to discriminate form.

\section{References}

Berkley, M. A., and J. M. Sprague (1979) Striate cortex and visual acuity functions in the cat. J. Comp. Neurol. 187: 679702.

Berman, N. (1977) Connections of the pretectum in the cat. J. Comp. Neurol. 174: 227-254.

Berman, N., and E. G. Jones (1977) A retino-pulvinar projection in the cat. Brain Res. 134: 237-248.

Berson, D. M., and A. M. Graybiel (1978) Parallel thalamic zones in the LP-pulvinar complex of the cat identified by their afferent and efferent connections. Brain Res. 147: 139148. 
Caldwell, R. B., and R. R. Mize (1981) Superior colliculus neurons which project to the cat lateral posterior nucleus have varying morphologies. J. Comp. Neurol. 203: 53-66.

Chalupa, L., M. J. Hughes, and R. W. Williams (1981) Receptive field properties in the tectorecipient zone of the cat's lateral posterior nucleus. Soc. Neurosci. Abstr. 7: 831.

Clare, M. S., and G. H. Bishop (1954) Responses from an association area secondarily activated from optic cortex. J. Neurophysiol. 17: 271-277.

Cowan, W. M., D. I. Gottlieb, A. E. Hendrickson, J. L. Price, and T. A. Woolsey (1972) The autoradiographic demonstration of axonal connections in the central nervous system. Brain Res. 37: 21-51.

de Olmos, J. S. (1977) An improved method for the study of central nervous connections. Exp. Brain Res. 29: 541-555.

Diamond, I. T. (1973) The evolution of the tectal-pulvinar system in mammals: Structural and behavioral studies of the visual system. Symp. Zool. Soc. Lond. 33: 205-233.

Diamond, I. T. (1976) Organization of the visual cortex: Comparative anatomical and behavioral studies. Fed. Proc. 35: 60-67.

Diamond, I. T., and W. C. Hall (1969) Evolution of neocortex. Science 164: 251-262.

Dreher, B., and A. J. Sefton (1979) Properties of neurons in cat's dorsal lateral geniculate nucleus: A comparison between medial interlaminar and laminated parts of the nucleus. J. Comp. Neurol. 183: 47-64.

Fujii, M., and N. Yoshii (1979) Hypothalamic projection to the pulvinar-LP complex in the cat: A study by the silver impregnation method. Neurosci. Lett. 12: 247-252.

Graham, J. (1977) An autoradiographic study of the efferent connections of the superior colliculus in the cat. J. Comp. Neurol. 173: 629-654.

Graybiel, A. M. (1972a) Some extrastriate visual pathways in the cat. Invest. Ophthalmol. 11: 322-333.

Graybicl, A. M. (1972b) Some ascending connections of the pulvinar and nucleus lateralis posterior of the thalamus in the cat. Brain Res. 44: 99-125.

Graybiel, A. M., and D. M. Berson (1980) Histochemical identification and afferent connections of subdivisions in the lateralis posterior-pulvinar complex and related thalamic nuclei in the cat. Neuroscience 5: 1175-1238.

Graybiel, A. M., and D. Berson (1981) On the relation between transthalamic and transcortical pathways in the visual system. In The Organization of the Cerebral Cortex, F. O. Schmitt, F. G. Worden, G. Adelman, and S. G. Dennis, eds., pp. 285-319, MIT Press, Cambridge, MA.

Guillery, R. W., E. E. Geisert, Jr., E. H. Polley, and C. A. Mason (1980) An analysis of the retinal afferents to the cat's medial interlaminar nucleus and to its rostral thalamic extension, the "geniculate wing." J. Comp. Neurol. 194: 117142.

Heath, C. J., and E. G. Jones (1971) The anatomical organization of the suprasylvian gyrus of the cat. Ergeb. Anat. Entwicklungsgesch. 45: 1-64.

Hoffman, K. -P. (1973) Conduction velocity in pathways from retina to superior colliculus in the cat: A correlation with receptive field properties. J. Neurophysiol. 36: 409-424.

Hoffman, K. -P., and A. Schoppmann (1975) Retinal input to direction selective cells in the nucleus tractus opticus of the cat. Brain Res. 99: 359-366.

Hollander, H., and H. Vanegas (1977) The projection from the lateral geniculate nucleus onto the visual cortex in the cat. A quantitative study with horseradish peroxidase. J. Comp. Neurol. 173: 519-556.

Hubel, D., and T. Wiesel (1969) Visual areas of the lateral suprasylvian gyrus (Clare Bishop area) of the cat. J. Physiol. (Lond.) 202: 251-260.
Hughes, H. C. (1980) Efferent organization of the cat pulvinar complex, with a note on bilateral claustrocortical and reticulocortical connections. J. Comp. Neurol. 193: 937-963.

Itoh, K., N. Mizuno, T. Suzimoto, S. Nomura, Y. Nakamura, and A. Konishi (1979) A cerebello-pulvino-cortical and retino-pulvino-cortical pathway in the cat as revealed by the use of the anterograde and retrograde transport of horseradish peroxidase. J. Comp. Neurol. 187: 349-358.

Itoh, K., M. Conley, and I. T. Diamond (1981) Different distributions of large and small retinal ganglion cells in the cat after HRP injections of single layers of the lateral geniculate body and superior colliculus. Brain Res. 207: 147-152.

Jones, E. G., and T. P. S. Powell (1971) An analysis of the posterior group of thalamic nuclei on the basis of its afferent connections. J. Comp. Neurol. 143: 185-216.

Kass, J. H. (1978) The organization of visual cortex in primates. In Sensory Systems of Primates, C. R. Noback, ed., pp. 151179, Plenum Press, New York.

Kaufman, E. F. S., A. C. Rosenquist, and D. Raczkowski (1982) The projections of single thalamic neurons onto multiple visual cortical areas in the cat. Invest. Ophthalmol. Vis. Sci. Suppl. 22: 47.

Kawamura, S., and E. Kobayashi (1975) Identification of laminar origin of some tectothalamic fibers in the cat. Brain Res. 91: 281-285.

Kawamura, S., J. M. Sprague, and K. Niimi (1974) Corticofugal projections from the visual cortices to the thalamus, pretectum, and superior colliculus in the cat. J. Comp. Neurol. 158: $339-362$.

Kawamura, S., N. Fukushima, and S. Hattori (1979) Topographical origin and ganglion cell type of the retino-pulvinar projection in the cat. Brain Res. 173: 419-429.

Kawamura, S., N. Fukushima, S. Hattori, and M. Kudo (1980) Laminar segregation of cells of origin of ascending projections from the superficial layers of the superior colliculus in the cat. Brain Res. 184: 486-490.

Kievet, J., and H. G. J. M. Kuypers (1977) Organization of the thalamocortical connexions to the frontal lobe in the rhesus monkey. Exp. Brain Res. 29: 299-322.

Killackey, H., M. Snyder, and I. T. Diamond (1971) Function of striatc and temporal cortex in the tree shrew. J. Comp. Physiol. Psychol. 74: 1-29.

Kuypers, H. G. J. M., M. Bentivoglio, C. E. Catsman-Berrevoets, and A. T. Bharos (1980) Double retrograde neuronal labelling through divergent axon collaterals, using two fluorescent tracers with the same excitation wave length which label different features of the cell. Exp. Brain Res. 40: 383392.

Lehmkuhle, S., K. E. Kratz, S. C Mangel, and S. M. Sherman (1980) Spatial and temporal sensitivity of X-and Y-cells in the dorsal lateral geniculate nucleus of the cat. J. Neurophysiol. 43: 520-541.

LeVay, S., and D. Ferster (1977) Relay cell classes in the lateral geniculate of the cat and the effects of visual deprivation. J. Comp. Neurol. 172: 563-584.

Leventhal, A. G., J. Keens, and I. Tork (1980) The afferent ganglion cells and cortical projections of the retinal recipient zone (RRZ) of the cat's "pulvinar complex." J. Comp. Neurol. 194: 535-554.

Maciewicz, R. J. (1975) Thalamic afferents to areas 17, 18 and 19 of cat cortex traced with horseradish peroxidase. Brain Res. 84: 308-312.

Marcotte, R. R., and B. V. Updyke (1981) Thalamic projections onto the visual areas of the middle suprasylvian sulcus in the cat. Anat. Rec. 199: 160A.

Marshall, W. H., S. A. Talbot, and H. W. Ades (1943) Cortical responses of the anesthetized cat to gross photic and electrical afferent stimulation. J. Neurophysiol. $6: 1-15$. 
Mason, R. (1978) Functional organization of the cat's pulvinar complex. Exp. Brain Res. 31: 51-66.

Morest, D. K. (1964) The neuronal architecture of the medial geniculate body of the cat. J. Anat. 98: 611-630.

Niimi, K., and E. Kuwahara (1973) The dorsal thalamus of the cat and comparison with monkey and man. J. Hirnforsch. 14: $303-325$.

Niimi, K., M. Kadota, and Y. Matsushia (1974) Cortical projections of the pulvinar nuclear group of the thalamus in the cat. Brain Behav. Evol. 9: 422-457.

Palmer, L. A., A. C. Rusenquist, and R. J. Tusa (1978) The retinotopic organization of the lateral suprasylvian areas in the cat. J. Comp. Neurol. 177: 237-256.

Raczkowski, D. (1979) Connections of the lateral posteriorpulvinar complex with the extrastriate visual cortex in the cat. Soc. Neurosci. Abstr. 5: 803.

Raczkowski, D., and A. C. Rosenquist (1980) Connections of the parvocellular $\mathrm{C}$ laminae of the dorsal lateral geniculate nucleus with the visual cortex in the cat. Brain Res. 199: 447-451.

Raczkowski, D., and $\Lambda$. C. Rosenquist (1981) Retinotopic organization in the cat lateral posterior-pulvinar complex. Brain Res. 221: 185-191.

Rioch, D., McK. (1929) Studies on the diencephalon of carnivora. I. The nuclear configuration of the thalamus, epithalamus, and hypothalamus of the dog and cat. J. Comp. Neurol. 49: 1-119.

Rodieck, R. (1979) Visual pathways. Annu. Rev. Neurosci. 2. 193-225.

Rose, J., and C. N. Woolsey (1949) Organization of the mammalian thalamus and the relationships to the cerebral cortex. Electroencephalogr. Clin. Neurophysiol. 1: 391-403.

Rosenquist, A. C., S. B. Edwards, and L. A. Palmer (1974) An autoradiographic study of the projections of the dorsal lateral geniculate nucleus and the posterior nucleus in the cat. Brain Res. 80: 71-93.

Rosenquist, A. C., D. Raczkowski, and L. L. Symonds (1982) Functional organization of the lateral posterior-pulvinar complex in the cat. In Changing Concepts of the Nervous System, A. Morrison and P. Strick, eds., pp. 261-279, Academic Press, Inc., New York.

Sanderson, K. J. (1971) The projection of the visual field to the lateral geniculate and medial interlaminar nuclei in the cat. J. Comp. Neurol. 143: 101-118.

Sherman, S. M. (1979) The functional significance of $X$ and $Y$ cells in normal and visually deprived cats. Trends Neurosci. 2: 192-195.

Sherman, S. M. (1982) Parallel pathways in the cat's geniculocortical system: W-, X-, and Y-cells. In Changing Concepts of the Nervous System, A. Morrison and P. Strick, eds., pp. 337-359, Academic Press, Inc., New York.

Sprague, J. M., J. Levy, A. DiBerardino, and G. Berlucchi
(1977) Visual cortical areas mediating form discrimination in the cat. J. Comp. Neurol. 172: 441-488.

Stanford, L. R., M. J. Friedlander, and S. M. Sherman (1981) Morphology of physiologically identified W-cells in the $\mathrm{C}$ laminae of the cat's lateral geniculate nucleus. J. Neurosci. 1: 578-584.

Stone, J., and B. Dreher (1973) Projections of X- and Y-cells of the cat's lateral geniculate nucleus to areas 17 and 18 of visual cortex. J. Neurophysiol. 36: 551-567.

Stone, J., B. Dreher, and A. Leventhal (1979) Hierarchical and parallel mechanisms in the organization of visual cortex. Brain Res. Rev. 1: 345-394.

Symonds, L. L., A. C. Rosenquist, S. B. Edwards, and L. A. Palmer (1981) Projections of the pulvinar-lateral posterior complex to visual cortical areas in the cat. Neuroscience 6 : 1995-2020.

Tong, L., R. Kalil, and P. Spear (1982) Thalamic projections to visual areas of the middle suprasylvian sulcus in the cat. J. Comp. Neurol. 212: 103-117.

Tusa, R. J., and L. A. Palmer (1980) Retinotopic organization of areas 20 and 21 in the cat. J. Comp. Neurol. 193: 147-164.

Tusa, R. J., L. A. Palmer, and A. C. Rosenquist (1978) The retinotopic organization of area 17 (striate cortex) in the cat. J. Comp. Neurol. 177: 213-236.

Tusa, R. J., A. C. Rosenquist, and L. A. Palmer (1979) Retinotopic organization of areas 18 and 19 in the cat. J. Comp. Neurol. 185: 657-678.

Tusa, R. J., L. A. Palmer, and A. C. Rosenquist (1981) Multiple cortical visual areas: Visual field topography in the cat. In Cortical Sensory Organization, C. N. Woolsey, ed., pp. 1-31, Humana Press, Clifton, NJ.

Updyke, B. V. (1977) Topographic organization of the projections from cortical areas 17,18 and 19 onto the thalamus, pretectum, and superior colliculus in the cat. J. Comp. Neurol. 173: 81-122.

Updyke, B. V. (1981) Projections from visual areas of the middle suprasylvian sulcus onto the lateral posterior complex and adjacent thalamic nuclei in cat. J. Comp. Neurol. 201: 477-506.

Van Essen, D. (1979) Visual areas of the mammalian cerebral cortex. Annu. Rev. Neurosci. 2: 227-263.

Wilson, P. D., M. H. Rowe, and J. Stone (1976) Properties of relay cells in the cat's lateral geniculate nucleus. A comparison of $\mathrm{W}$-cells with $\mathrm{X}$ - and Y-cells. J. Neurophysiol. 39. 1193-1209.

Winer, J. A., I. T. Diamond, and D. Raczkowski (1977) Subdivisions of the auditory cortex of the cat: The retrograde transport of horseradish peroxidase to the medial geniculate body and posterior thalamic nuclei. J. Comp. Neurol. 157: 387-418.

Woolsey, C. N. (1961) Organization of cortical auditory system. In Principles of Sensory Communication, W. A. Rosenblith, ed., pp. 235-257, MIT Press, Cambridge, MA. 